\title{
Les cours d'eau dans les incantations chamaniques des Indiens yucuna (Amazonie colombienne)
}

\section{Laurent Fontaine}

\section{(2) OpenEdition \\ 1 Journals}

Édition électronique

URL : http://journals.openedition.org/jsa/11693

DOI : 10.4000/jsa. 11693

ISSN : 1957-7842

Éditeur

Société des américanistes

Édition imprimée

Date de publication : 5 octobre 2011

Pagination : 119-149

ISSN : 0037-9174

\section{Référence électronique}

Laurent Fontaine, «Les cours d'eau dans les incantations chamaniques des Indiens yucuna

(Amazonie colombienne) », Journal de la société des américanistes [En ligne], 97-1 | 2011, mis en ligne le 10 décembre 2014, consulté le 19 avril 2019. URL : http://journals.openedition.org/jsa/11693 ; DOI

10.4000/jsa. 11693 


\title{
LES COURS D'EAU \\ DANS LES INCANTATIONS CHAMANIQUES DES INDIENS YUCUNA (AMAZONIE COLOMBIENNE)
}

\author{
Laurent FONTAINE *
}

\begin{abstract}
Chez les Yucuna, chaque cours d'eau a une histoire, des maîtres ou des habitants d'origine. Tous sont mentionnés dans des invocations, appelées juní maná (« invocations de l'eau »). Ces invocations énumératives sont fondamentales dans le chamanisme yucuna : elles constituent une structure de base reprise dans de nombreuses incantations, lesquelles sont complétées en fonction du type de cure à prodiguer. Elles rappellent, expliquent et situent l'origine de chaque élément naturel en l'associant souvent à un extrait d'histoire ou de récit mythique bien précis, envisagé en fonction de ses conséquences dans la vie quotidienne. Nous présentons ici ces « invocations de l'eau » dans leur langue d'origine ainsi que les commentaires du thérapeute. [Mots-clés : incantation, chamanisme, Yucuna, Amazonie, Colombie.]
\end{abstract}

Rivers in shamanic incantations of Yucuna Indians (Colombian Amazon). For Yucuna Indians, every river has its history, its masters or original inhabitants. They are mentioned in invocations called juní maná ( invocations of water »). These invocations are one of fundamentals of the shamanism of the Yucuna: they constitute a basic structure repeated in many incantations, which are supplemented with other invocations depending on the type of treatment to be provided. They recall, explain and situate the origin of every natural element referring to some particular extract of history or myth, considered with its consequences in everyday life. In this text, the "invocations of water $»$ are presented in their original language with the comments of the therapist. [Key words : incantation, shamanism, Yucuna, Amazon, Colombia.]

Los ríos en las oraciones chamánicas de los indígenas yucuna (Amazonía colombiana). Para los yucuna, cada río tiene su historia, sus maestros o sus habitantes originales. Todos son mencionados en unas invocaciones llamadas juní maná (« invocaciones del agua »). Estas invocaciones son fundamentales en el chamanismo yucuna : constituyen una estructura básica utilizada en muchas oraciones chamánicas, las cuales son complementadas según la curación que se hace. Recuerdan, explican y localizan el origen de

* Laboratoire des langues et civilisations à tradition orale (Lacito, UMR 7107 du CNRS), 7 rue Guy Môquet (bâtiment D), 94801 Villejuif cedex [fontaine@vjf.cnrs.fr].

Journal de la Société des Américanistes, 2011, 97-1, pp. 119-149. (C) Société des Américanistes. 
cada elemento natural asociándolo a menudo con una parte específica de historia o de mito, considerada con sus consecuencias en la vida cotidiana. Aquí se presentan las « invocaciones del agua » juntas con los comentarios del curandero. [Palabras claves : oración, chamanismo, yucuna, Amazonía, Colombia.]

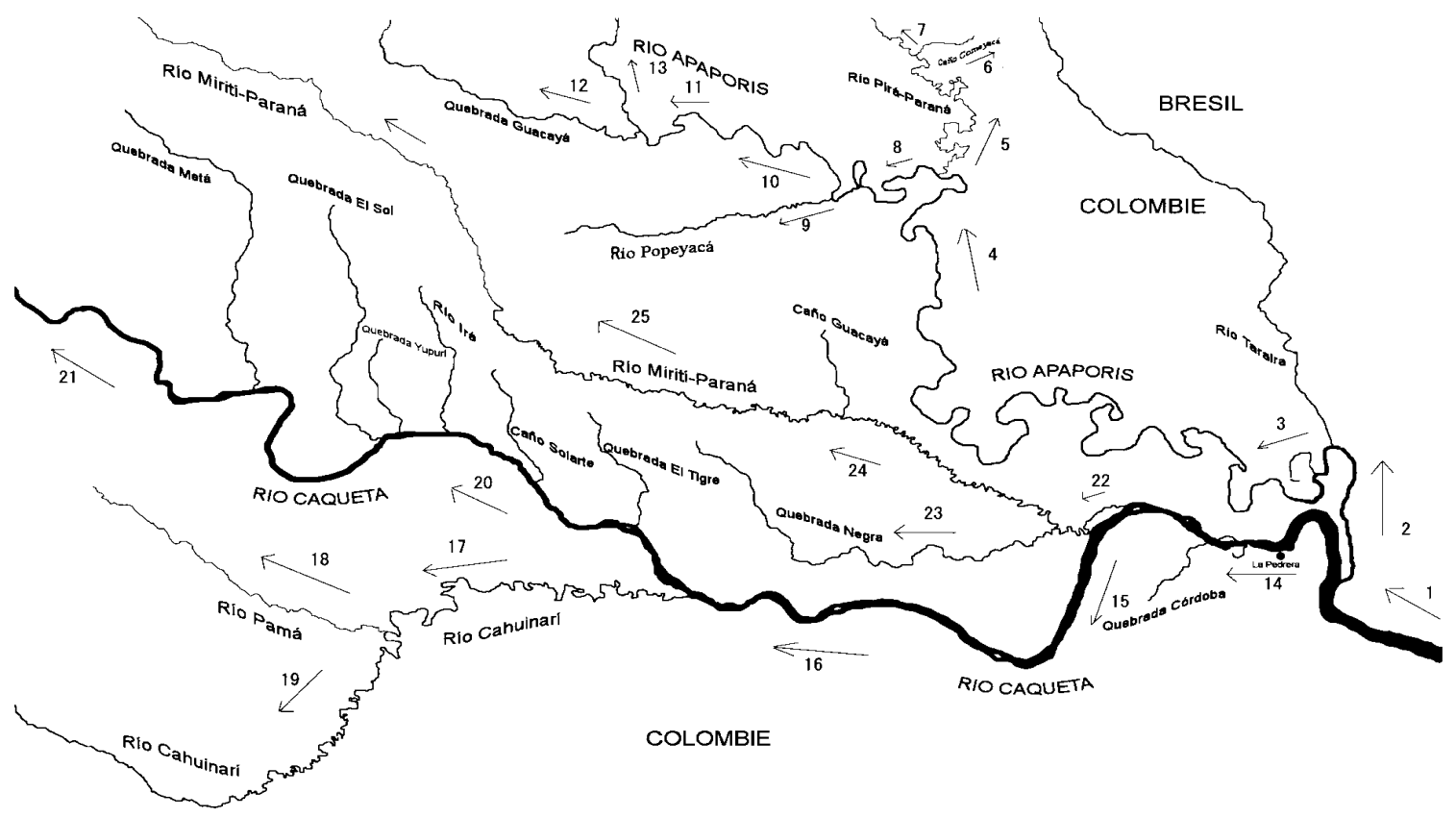

FIG. 1 - Le cheminement de l'invocation de l'eau.

Dans une société sans écriture et sans iconographie de l'espace (Figure 1) comme celle des Indiens yucuna ${ }^{1}$ d'Amazonie colombienne, on peut se demander comment ces derniers peuvent parcourir de longues distances au sein de leur milieu naturel, en sachant s'orienter et en anticipant ce qu'ils vont y trouver. En étudiant les incantations de plusieurs soigneurs yucuna, nous avons pu nous rendre compte que les incantations chamaniques de ces Indiens ne sont pas uniquement composées de " paroles magiques » censées avoir des effets sur le monde, mais qu'elles constituent également un immense domaine de connaissance du territoire, en relation avec la faune et la flore. L'étude de ces incantations montre que leur connaissance s'étend bien au-delà du domaine de la chasse et de 
la pêche, de la cueillette et de l'horticulture de chaque famille, et qu'elles sont extrêmement utiles pour comprendre les rapports que les Yucuna entretiennent avec leur milieu naturel ${ }^{2}$. En effet, elles constituent une manière d'organiser et d'ordonner une gigantesque accumulation de savoirs, grâce à leur haut niveau de structuration permettant de se référer au monde dans toute sa complexité.

Nous proposons de présenter dans leur langue d'origine les « invocations de l'eau » des Yucuna, ainsi que les commentaires du thérapeute, Mario Matapi, qui a accepté de nous les exposer ${ }^{3}$. L'ensemble fut recueilli fin février 2008. Ces incantations constituent normalement un langage secret qu'il est rare de pouvoir recueillir, même avec les autorisations nécessaires ${ }^{4}$. En langue yucuna, l'invocation étudiée est nommée juní maná (« appel de l'eau »). Elle ne forme jamais à elle seule une incantation complète ; elle est toujours dirigée vers un objectif particulier. Elle ne constitue que l'un des éléments fondamentaux de beaucoup d'incantations, surtout parmi les plus longues. Ces dernières sont généralement considérées comme très difficiles à mémoriser et à maîtriser compte tenu de leur durée (parfois plus de douze heures) et des risques encourus pour celui qui les prononce, ainsi que pour ses proches, en particulier si l'incantation est mal préparée (manque de coca à mâcher) ou mal formulée (oubli de certains ancêtres ou esprits importants). Les invocations de l'eau sont utilisées partiellement ou intégralement dans différents types d'incantations : celles destinées au développement d'un enfant en bas âge, censées le protéger et lui permettre d'absorber toutes sortes de nourritures ; celles destinées à son développement initiatique et chamanique, requises pour la mémorisation des paroles rituelles (mythes, chants, incantations, dialogues cérémoniels) ; celles destinées à la neutralisation des effets potentiellement nuisibles des aliments, pour clore une diète, particulièrement lorsque les adolescents terminent le rite de Yuruparí ${ }^{5}$; les incantations annuelles (reproduites chaque année) ayant pour but d'éviter et de prévenir les dangers de la nature se répétant spécifiquement à chaque nouvelle saison (Fontaine 2010a).

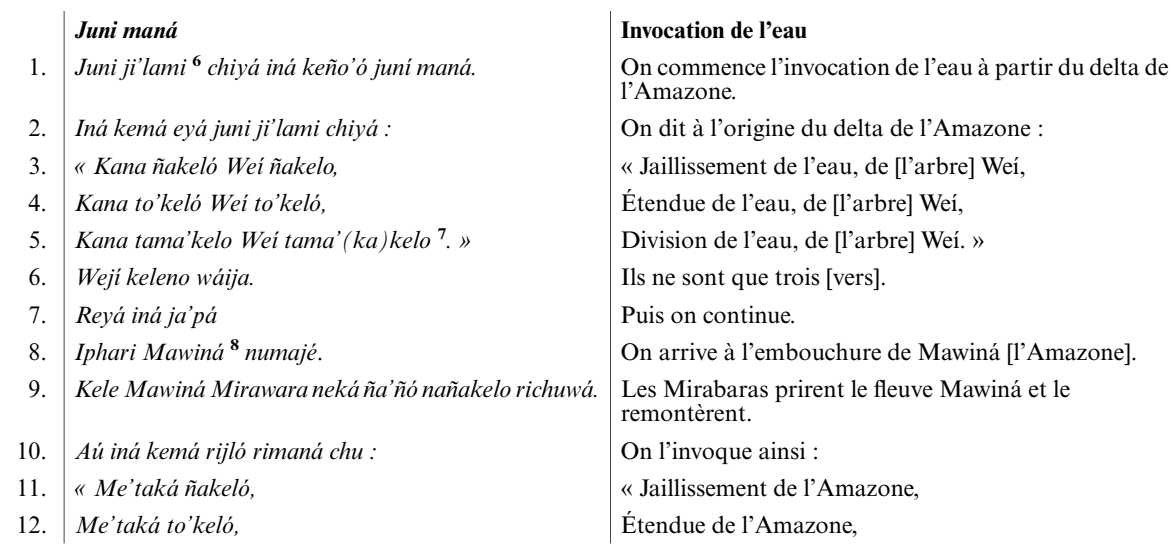




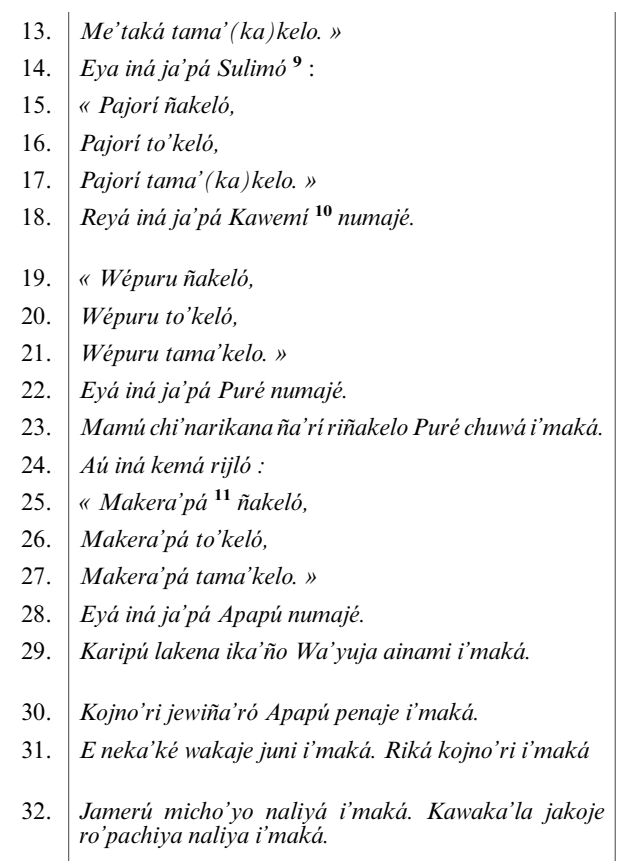

Division de l'Amazone. »

Puis l'on continue jusqu'au fleuve Sulimó [Solimões] :

« Jaillissement du Solimões,

Étendue du Solimões,

Division du Solimões. »

De là, on poursuit jusqu'à l'embouchure du Kawemí [Caquetá].

« Jaillissement du Caquetá,

Étendue du Caquetá,

Division du Caquetá. »

On continue jusqu'à l'embouchure du Puré.

La divinité Sábalo remonta le fleuve Puré.

On lui dit :

« Jaillissement du Puré,

Étendue du Puré,

Division du Puré. »

On poursuit jusqu'à l'embouchure de l'Apaporis.

C'est là que les Karipú lakena abattirent l'arbre patriarche Wa'yuja ${ }^{12}$.

En tombant, il devint l'Apaporis.

L'eau vient de ce jour où ils l'abattirent. Et l'arbre bascula.

Comme Jamerú ne voulait pas leur donner l'eau qu'elle cachait à l'intérieur, elle le [l'arbre] fit passer à travers la terre ${ }^{13}$.

C'est pourquoi nous disons que cette mère de l'eau est si puissante.

De là, l'Apaporis s'est divisé.

Alors on lui dit en termes invocatoires :

« Jaillissement des Karipú lakena,

Étendue des Karipú lakena,

Division des Karipú lakena. »

« Jaillissement de l'Eau [qui fut] dissimulée,

Étendue de l'Eau [qui fut] dissimulée,

Division de l'Eau [qui fut] dissimulée. »

De là, on se sépare [bifurque] plus haut sur l'A paporis.

De l'embouchure de l'Apaporis, on continue jusqu'à celle de Taraira.

Comme la divinité Taraira [un poisson] remonta cet affluent [pour aller s'installer], on l'appela Taraira.

Les poissons taraira y abondent.

Alors on lui dit :

« Jaillissement de la fosse de Taraira,

Étendue de la fosse de Taraira,

Division de la fosse de Taraira. »

De là on le remonte jusqu'en haut.

$\mathrm{Au}$ bord des rives, on trouve alors des palmeraies d'aguaje qui furent des grands ancêtres Yaúna.

Ils s'appellent Ne'ekawá et Ne'ewíruwa. 
53. Aú pimá reyá junijlo :

54. «Ne'ekawá ñakeló,

55. Ne'ekawá to'keló,

56. Ne'ekawá tama' (ka)kelo. »

57. «Ne'ewiruwa ñakeló,

58. Ne'ewiruwa to'keló,

59. Ne'ewíruwa tama'(ka)kelo. »

60. Eyá iná pa'ó iná yámojo.

61. Ja'pari ero'jopaya numaya Kariyaka ${ }^{17}$ numaje.

62. « Makariyárena ñakeló,

63. Makariyárena to'keló,

64. Makariyárena tama'kelo. »

65. Eyá iná ja’pá Pirá numaje.

66. Kari ña'ri riñakelo rinumaya.

67. Ina'uké chi'narikana ke ri'maká.

68. « Kari ñakeló

69. Kari to'keló,

70. Kari tama'kelo. ”

71. Richuwa yenojo iná i'jná yenojo Komeyaká numaje.

91. Letua chi'ná ña'ñó nañakeló Po'peyá chuwá.

92. Piyute nachi'narikana, wachi'narikana najwa'té.

93. Aú iná kemá Po'peyajlo :

94. « Makuwachi ${ }^{21}$ iwamá ñakeló,
À l'eau de là-bas, on dit alors :

« Jaillissement de Ne'ekawá,

Étendue de Ne'ekawá,

Division de Ne'ekawá. »

« Jaillissement de Ne'ewíruwa,

Étendue de Ne'ewíruwa,

Division de Ne'ewíruwa. »

Puis l'on redescend.

On passe l'embouchure de Taraira, puis on arrive à celle du lac Kariyaka «sauce tucupi » [Caparú].

« Jaillissement de Kariyaka,

Étendue de Kariyaka,

Division de Kariyaka. »

On continue jusqu'à l'embouchure du Pirá-Paraná.

Kari remonta ce fleuve [pour aller y vivre].

Il était un grand ancêtre des humains.

« Jaillissement de Kari,

Étendue de Kari,

Division de Kari. »

En le remontant, on arrive à l'embouchure du Komeyaká [Caño Comeyacá].

Un grand ancêtre makuna [qui était une tortue terrestre] le remonta pour aller s'y installer.

Alors on lui dit :

« Jaillissement des enfants de la tortue,

Étendue des enfants de la tortue,

Division des enfants de la tortue. »

On passe [l'embouchure du Comeyacá] pour aller jusqu'en haut [du Pirá-Paraná].

C'est en haut que la divinité toucan alla s'installer.

Alors on lui dit :

« Jaillissement des enfants du toucan,

Étendue des enfants du toucan,

Division des enfants du toucan. »

Puis l'on poursuit jusqu'à sa source.

L'ancêtre du « laurier » prit pour lui cet endroit.

Alors on invoque ainsi l'eau qui s'y trouve :

« Jaillissement du laurier,

Étendue du laurier,

Division du laurier. »

Puis l'on revient en arrière jusqu'à l'embouchure.

En traversant pour passer de l'autre côté du fleuve, on arrive à l'embouchure du Po'peyá [río Popeyacá].

Les ancêtres des Letuama prirent cette rivière.

Nous avons en commun avec eux un même grand ancêtre anaconda.

Alors on dit au Popeyacá :

« Jaillissement du milieu de Makuwachi, 


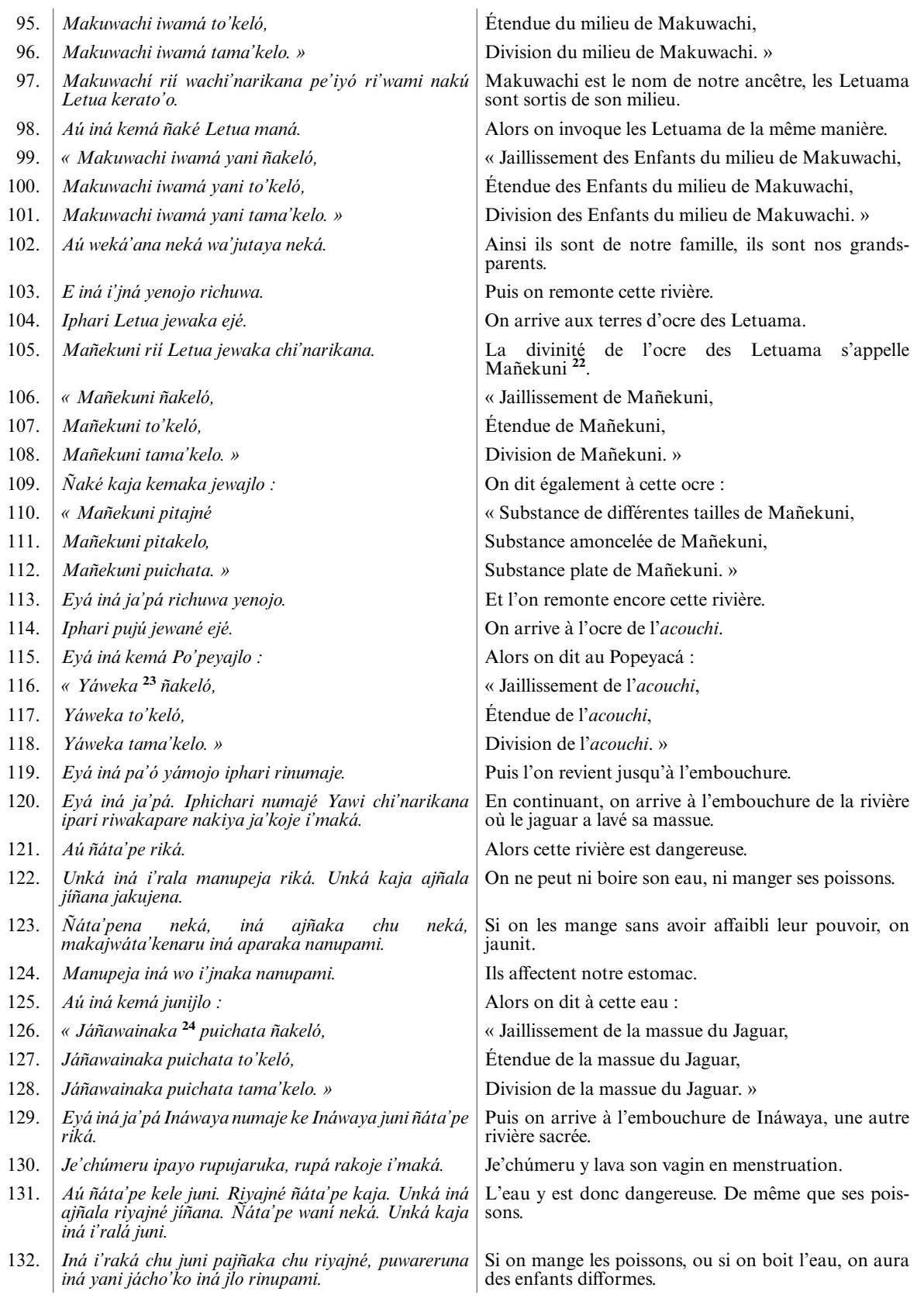


133. Jiñana ra’kojena ne’iyajena chu'takeja neká panakojechaka. Iyama ta najipí, iyamala newilaru.

134. Re’iyaje mejluru, re’iyajé unká takú aphú i’malá, re’iyaje manumaru, ke nanupami iná ajñaka chu jínana rakiyajena.

135.

Aú iná kemá rimaná chu rijló :

136. « Je’chúmeru pujaruka ñakeló,

137.

138.

139.

140.

57. Ñaké iná amaka chu jiñañana aú iná kemá : «Ja'yukumu. Kajrú nojena waní nuji'chá jiñañana. Ijmuláruna waní.

158. Kajrú najilá nawó chu. ”

159. Ejechami iná ña'ká ijmuláruna jiñañana kejiláuna.

160. Ne'raka chu iná, unká iná kemala : « Jiñañá i’richari nuká!"

161. Aú iná kemá: "Ja’yukumu. Pácho’ta pa’napitá nojló. "

162. E unká ne’rala iná ke nanakoje re’iyajena jiñañana.

163. Re kaja Karipú lakena kátaño pajwa'teka re. Natewíyokako re i'maká.
Certains poissons sont collés entre eux. D'autres on deux queues, ou deux têtes.

D'autres encore n'ont pas d'yeux, pas de narines, ou pas de bouche, et ainsi sera la malédiction pour ceux qui mangent les poissons de cette rivière.

Alors on s'adresse ainsi à cette rivière :

« Jaillissement du vagin en menstruation de Je'chúmeru,

Étendue du vagin en menstruation de Je'chúmeru,

Division du vagin en menstruation de Je'chúmeru. »

Puis l'on continue jusqu'à la marmite à poissons-chats. Il y a un gros rocher.

C'est là que les Karipú lakena firent leur marmite à poissons-chats.

C'est là qu'ils allaient les pêcher.

Là, il y a un palmier aguaje [appartenant au monde spirituel].

Quand l'aguaje charge ses fruits, les poissons-chats abondent là où ils tombent dans la rivière.

$\mathrm{Si}$, après les avoir mangés, on a la diarrhée, il ne faut pas dire : « ces poissons m’ont donné la chiasse ».

Cela empirerait.

Quand ils commencent à nous donner la diarrhée, l'on dit [comme les Karipú lakena] :

«Frère. J'ai mal supporté cette huile.

Je crotte vraiment trop dur. »

Et la diarrhée se termine.

Quand ces poissons pourrissent : on ne dit pas « mes poissons sont avariés »".

Sinon ils vont pourrir encore plus.

Il faut dire : «Frère. Mes poissons sont vraiment bien boucanés.

Et ils vont sécher. »

Si l'on en pêche des petits, il ne faut pas dire : « J'ai pris des poissons trop petits $\gg$.

Sinon ils vont se rapetisser encore davantage, et même devenir squelettiques.

En regardant de tels poissons-chats, il faut dire :

«Frère. Ils sont beaux et gros, ces poissons que j'ai attrapés.

Et ils deviennent déjà plus gras. "

Après cela on attrape des poissons-chats plus gros et plus gras.

Lorsqu'ils nous piquent [de leurs nageoires], on ne dit pas : «Un poisson-chat m'a piqué !»

Il faut dire : «Frère. Ce poisson a levé les bras pour moi » ${ }^{26}$.

Et on ne sent plus leur piqûre.

C'est là [sur le rocher] que les Karipú lakena jouèrent à lutter [avec les vagues qui les frappent]. 
164. «Maare wakátajo, yuríjero walakena i’majikaño maare imajikañojlo penaje riyurojo i’majika. "

165.

166.

167.

168.

169.

170.

171. Kaphí riphaka iná nakú iná ika'ka jwilá ke rijña'a iná rinakuwa."

172. Eyá ripata iná. Erika'a iná wichá Apapú ja’kojé. Sujwí inája'kójunapejé.

173.

Iná amaka chu ñaké ra’kó junapejé, unká iná kemalá "rikapi"choke".

174.

198. Jañunaké yani to’keló,

chojé apú juni nakiyana i'maká, riká rujláma'a rejé i’maká Yapiyá penaje.

Kajrú nojena po’jona rakú, mamuna rakú, manupamiruna neká.

Unká ñáta'pe kalé Yapiyá ya'jné pheñáwilá kemajika rinaku.

Aú iná kemá kele Yapiyajlo .

Wana ${ }^{28}$ chojé romo'ó juni i'maká.

" Jíwaru ${ }^{29}$ puichaku ñakeló,

Jiwaru puichaku to'keló,

Jiwaru puichaku tama'kelo. »

« Jíwa náneru ${ }^{30}$ ñakeló,

Jiwa náneru to’keló,

Jíwa náneru tama'kelo. »

Reyá iná i’jná richuwa yenojó. Iphari Kunami numaje.

Kunami Jatímaja ña'ñó nañakelo richuwá.

Aú iná kemá Kunámijlo rinumaya :

Jañunaké yani ñakeló [ils dirent :] « Ici nous allons faire un jeu, qui restera pour nos descendants. »

Lajmuchí dit à son frère : « Frère. Attends-moi ici.

Nous allons montrer notre force. »

Il plongea dans l'eau.

Puis il ajouta : «Nous allons nous mesurer l'un à l'autre.

Je veux que tu viennes me rentrer dedans en te tenant solidement sur tes jambes.

Les vagues vont venir sur nous.

Quand elles arrivent, il faut en profiter pour frapper l'autre ${ }^{27}$. "

Jusqu'à ce qu'une vague emporte l'un des deux qui, en tombant au milieu des rapides de l'Apaporis, disparaîtra sous les eaux.

Quand on voit ce dernier tomber à l'eau, il ne faut pas dire : « il a disparu ».

Mais « il est parti vivre dans le monde des profondeurs. » Ainsi il [le maître du lieu] le laissera ressortir de l'eau avant qu'il ne se noie.

Alors l'on s'adresse ainsi à l'eau de l'Apaporis :

« Jaillissement de la marmite des Karipú lakena,

Étendue de la marmite des Karipú lakena,

Division de la marmite des Karipú lakena. »

Et l'on continue jusqu'à l'embouchure du Yapiyá [Quebrada Guacayá].

L'eau du Yapiyá est transparente, sa couleur est bleu clair.

C'est Je'chúmeru qui, en allant puiser de l'eau, en renversa à cet endroit qui devint le Yapiyá.

Riká rujláma'a rejé i’maká.
On y trouve beaucoup de sabaletas et sábalos, qui ceux-là sont inoffensifs.

Ils peuvent être mangés sans danger, disaient les anciens.

Alors on dit au Yapiyá :

Eau qu'elle puisa avec un bambou.

Ce qu'elle renversa.

« Jaillissement du bambou évidé de Je'chúmeru,

Étendue du bambou évidé de Je'chúmeru,

Division du bambou évidé de Je'chúmeru. »

« Jaillissement de Je'chúmeru,

Étendue de Je'chúmeru,

Division de Je'chúmeru. »

Puis l'on remonte cette rivière jusqu'à l'embouchure du Kunami ${ }^{31}$.

C'est cette rivière que les premiers ancêtres des Jatímaja ${ }^{32}$ prirent pour s'installer.

On dit alors à cette rivière Kunami :

« Jaillissement des descendants du jaguar Iyánuna,

Étendue des descendants du jaguar Iyánuna, 


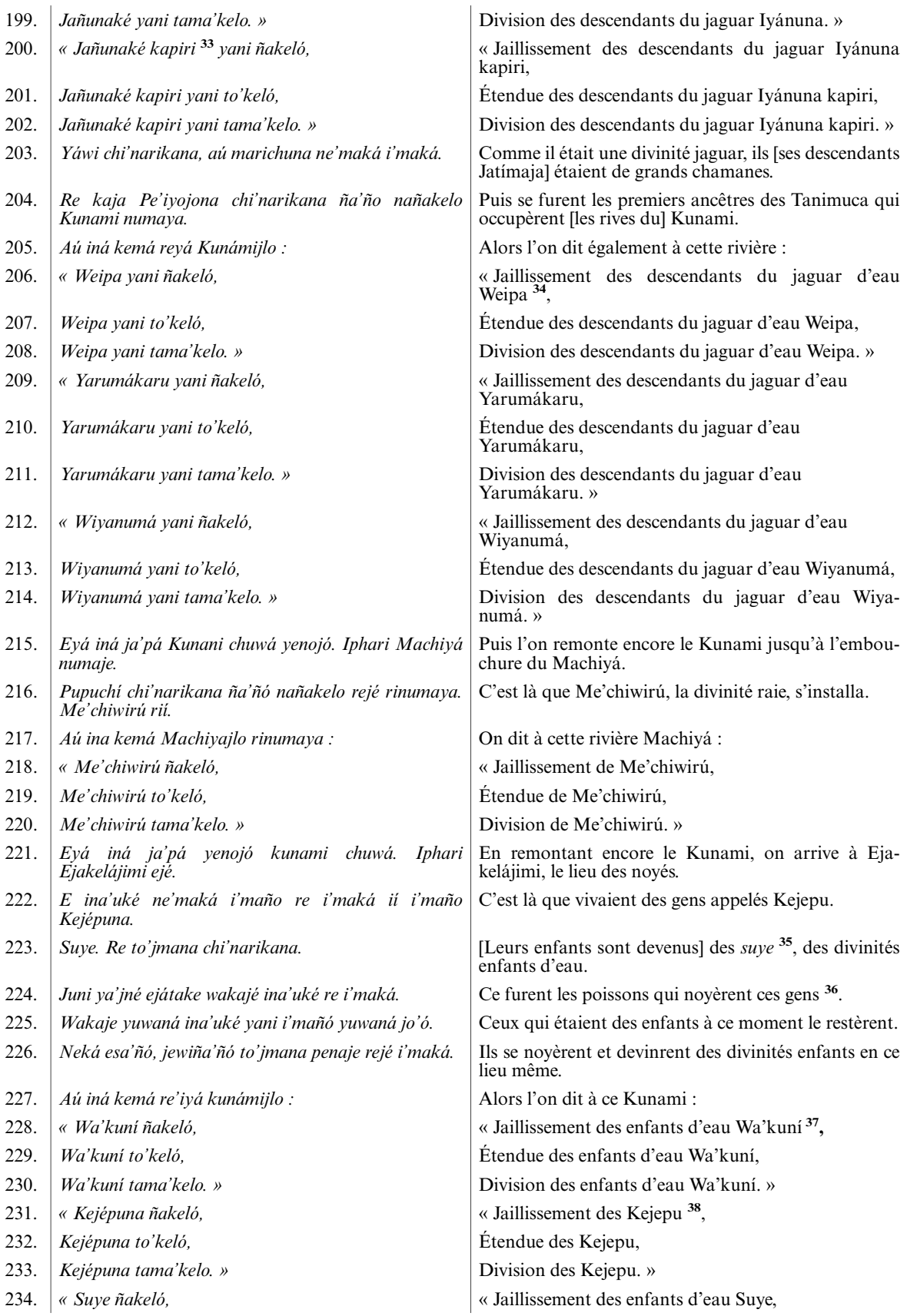




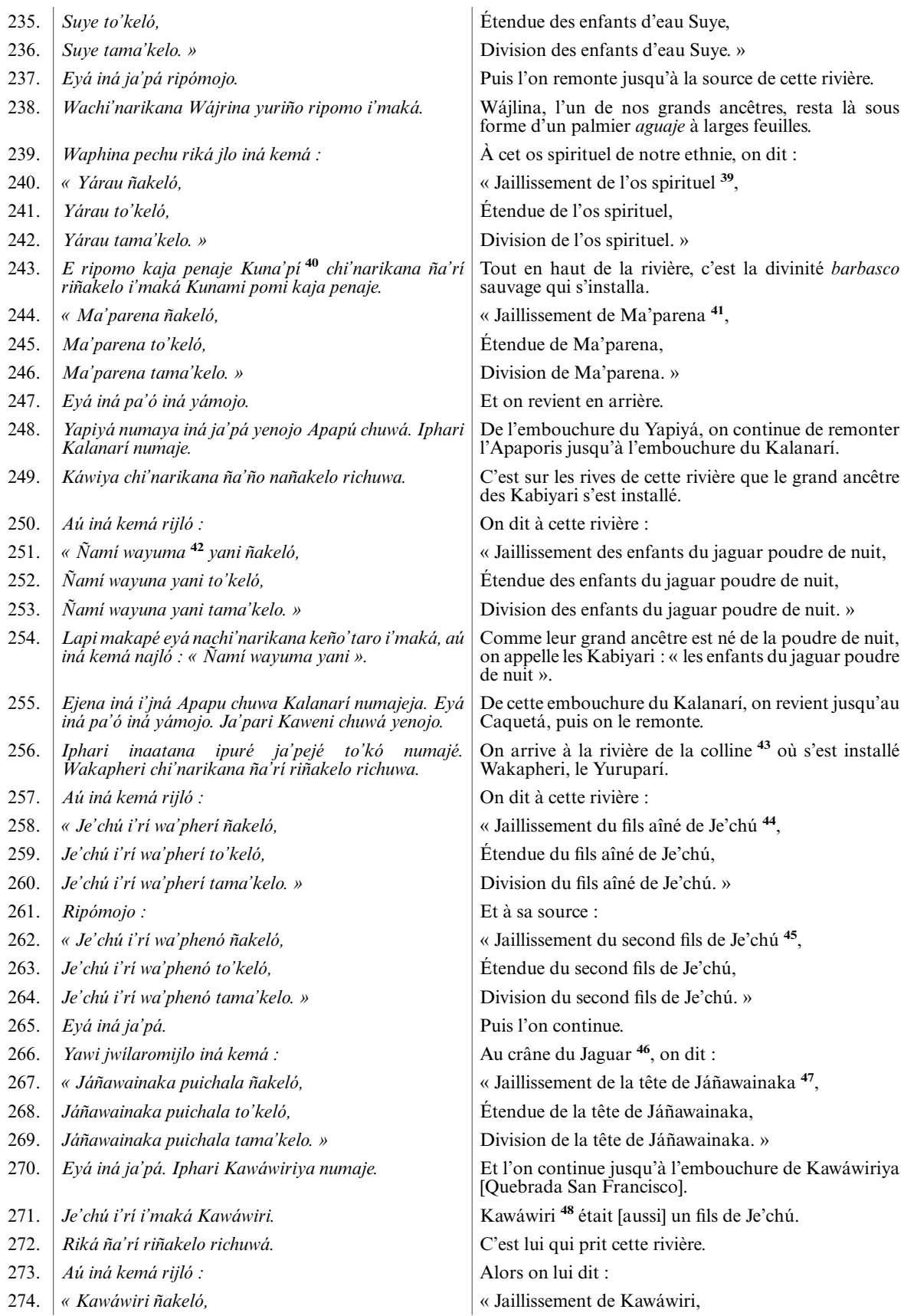




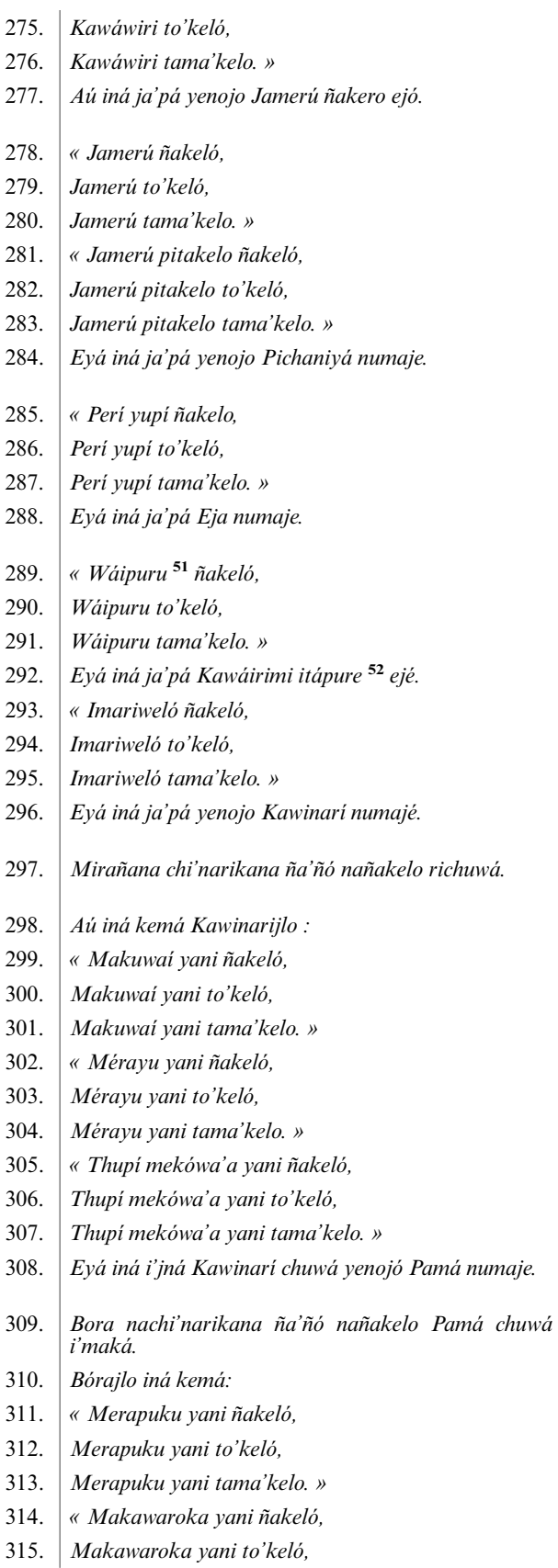

Étendue de Kawáwiri,

Division de Kawáwiri. »

Puis l'on remonte jusqu'à la demeure de Jamerú ${ }^{49}$ [les rapides de Córdoba].

« Jaillissement de Jamerú,

Étendue de Jamerú,

Division de Jamerú. »

« Jaillissement de l'amoncellement de Jamerú,

Étendue de l'amoncellement de Jamerú,

Division de l'amoncellement de Jamerú. »

On poursuit jusqu'à l'embouchure de Pichaniyá, la rivière de Tonnerre [Quebrada Córdoba].

« Jaillissement du maître du curare ${ }^{\mathbf{5 0}}$,

Étendue du maître du curare,

Division du maître du curare. »

Puis l'on poursuit jusqu'à l'embouchure du MiritíParaná.

« Jaillissement de l'ancêtre des pierres de foudre,

Étendue de l'ancêtre des pierres de foudre,

Division de l'ancêtre des pierres de foudre. »

Puis l'on continue jusqu'au Raccourci de Kawáirimi.

« Jaillissement de Je'chúmeru Imariweló ${ }^{\mathbf{5 3}}$,

Étendue de Je'chúmeru Imariweló,

Division de Je'chúmeru Imariweló. »

On continue jusqu'à l'embouchure du Kawinarí [río Cahuinarí].

Ce sont les premiers ancêtres des Miraña qui occupèrent cette rivière.

Alors on dit au Kawinarí :

" Jaillissement des enfants de Makuwaí ${ }^{\mathbf{4}}$,

Étendue des enfants de Makuwaí,

Division des enfants de Makuwaí. »

« Jaillissement des enfants de Mérayu,

Étendue des enfants de Mérayu,

Division des enfants de Mérayu. »

« Jaillissement des enfants du coco ${ }^{55}$

Étendue des enfants du coco,

Division des enfants du coco. »

Puis l'on remonte le Kawinarí jusqu'à l'embouchure du Pamá [río Pamá].

Les premiers ancêtres des Bora se sont installés le long de cette rivière.

À ces Bora, l'on dit :

« Jaillissement des enfants de Merapuku,

Étendue des enfants de Merapuku,

Division des enfants de Merapuku. »

"Jaillissement des enfants de Makawaroka,

Étendue des enfants de Makawaroka, 
316.

317.

318.
Makawaroka yani tama'kelo. 》

Eyá iná pa’ó iná yámojo.

Kawinari numaya iná ja'pá Uphé numajé.

Perí chi'narikana ña'rí riñakelo richuwá i’maká

Aú iná kemarí Uphejlo :

« Juni numari ${ }^{\mathbf{5 6}}$ ñakeló,

Juni numari to'keló,

Juni numari tama'kelo. »

Uphé chuwá iná i’jná yenojo.

Re a'pá Peri chi'narikana jala'kari ja'pá Uphé wa'pojó i’maká. Karipú lakena noke wakaje ri'maká kapichiri aú.

Wakajé riká inami rala'ká ja'pá inami Uphé wa’pojó i’maká. Kaja penaje ka ja'pá yuriro Uphé wa'pojó. Aú iná kemá ñaké rijló :

« Juni numari jipaji ${ }^{\mathbf{5 8}}$ ñakeló,

Juni numari jipají to'keló,

Juni numari jipají tama'kelo. »

Eyá iná ja’pá yenojo ripómojo.

Jarechina ña'ñó nañakelo ripómojo.

Aú iná kemá rijló :

«Lainúiri ñakeló,

Lainúiri to’keló,

Lainúiri tama'kelo. »

Perikana la'yana chi'narikanajlo iná kemá :

"Perikana yani ñakeló,

Perikana yani to'keló,

Perikana yani tama'kelo. "

Ripomo kaja penaje :

" Kuyerí yani ñakeló,

Kuyerí yani to'keló,

Kuyerí yani tama'kelo. "

Kele jarechina yuriño Uphé pomo i’maká.

Aú kajrú eja'wá minaná yuriko rejó, Uphé pomo.

Eyá iná pa’ó iná yámojo.

Uphé numaya iná ja'pá Yanayá numajé.

Pá'yumi ña'rí riñakelo richuwa napu riká.

Aú iná kemá Yanayajlo:

"Wapheno ${ }^{62}$ ñakeló,

Wapheno to'keló,

Wapheno tama'kelo. "
Division des enfants de Makawaroka. »

Après, on redescend.

De l'embouchure du Kawinarí, on continue jusqu'à celle de Uphé [Quebrada El Tigre].

La divinité aigle harpie prit cette rivière.

Alors on dit à Uphé :

« Jaillissement de l'aigle venu de la mer,

Étendue de l'aigle venu de la mer,

Division de l'aigle venu de la mer. »

Puis l'on remonte cette rivière Uphé.

Sur ses rives, on trouve des plantes à curare, car c'est là que l'aigle urina lorsqu'il se fit toucher par la flèche empoisonnée des Karipú lakena ${ }^{57}$.

Comme son urine était affectée par leur curare, des plantes à curare finirent par pousser à cet endroit.

Alors on lui dit également :

« Jaillissement de l'urine de l'aigle venu de la mer,

Étendue de l'urine de l'aigle venu de la mer,

Division de l'urine de l'aigle venu de la mer. »

Puis l'on remonte le haut de la rivière.

Les esprits telluriques Jarechina prirent le haut de la rivière.

Alors on dit à cette rivière :

« Jaillissement de Lainúiri ${ }^{\mathbf{5 9}}$,

Étendue de Lainúiri,

Division de Lainúiri. "

En nommant le grand ancêtre tellurique La'yana, on dit :

« Jaillissement des enfants de Perikana ${ }^{\mathbf{6 0}}$,

Étendue des enfants de Perikana,

Division des enfants de Perikana. ”

Enfin, à la source de la rivière :

«Jaillissement des enfants de Kuyerí ${ }^{61}$,

Étendue des enfants de Kuyerí,

Division des enfants de Kuyerí. »

Tels sont les esprits telluriques qui s'installèrent en haut de la rivière Uphé.

Ainsi, le haut de cette rivière est peuplé de nombreux maîtres de la forêt.

Puis l'on redescend.

De l'embouchure du Uphé, on continue jusqu'à celle du Yanayá [Caño Solarte].

Pá'yumi, le maître de l'eau s'y installa.

Alors on dit au Yanayá :

« Jaillissement de Wapheno,

Étendue de Wapheno,

Division de Wapheno. " 
354. Kele Yanayá chuwá Pä'yumi ${ }^{63}$ apu jo'rí Ko'rena $\mid$ numajé ejá chojé.

355.

Yanayá numaya iná ja'pá U'wiyá ${ }^{\mathbf{6 4}}$ numajé.

356.

Yanawirí ña'rí riñakelo richuwa i'maká rinumaya.

357. «Yanawirí ñakeló,

358. Yanawiri to'keló,

359. Yanawiri tama'kelo. "

360. Reyá iná ja’ pá Kúmuka numaje.

361. " Makumuyá ñakeló,

362. Makumuyá to'keló,

363. Makumuyá tama'kelo. »

364. Piyute riká re kumu ripechu.
Kúmuka numaya iná ja'pá Irá numaje.

« Kawanakú ñakeló,

Kawanakú to'keló,

Kawanakú tama'kelo. "

Eyá iná ja’pá yenojo Irá chuwá Mújala numaje.

"Ma'riweló ñakeló,

Ma'riweló to'keló,

Ma'riweló tama'kelo. »

Eyá iná ja'pá Wayuriya numaje.

" Ma'ínaniru ñakeló,

Ma'ínaniru to'keló,

Ma'ínaniru tama'kelo. "

Eyá iná ja’pá Jiñana aló ejé.

" Junirú ñakeló,

Junirú to'keló,

Junirú tama'kelo. »

" Junirú weló ñakeló,

Junirú weló to'keló,

Junirú weló tama'kelo. "

Eyá iná ja’pá Irá numaya Ipatuya numaje.

« Makuwapá ñakeló,

Makuwapá to'keló,

Makuwapá tama'kelo. »

Eyá iná ja’pá Waniyá numaje.

Je'chúmeru ipayo rujimá chiya ruwijune rakoje i'maká.

2. Aú ipurala’ni waniyá.
Sur le Yanayá, il y a le passage de Páyumi, que ce dernier creusa pour aller jusqu'à l'embouchure du Ko'rena sur le Mirití-Parana.

De l'embouchure du Yanayá, on continue jusqu'à celle de la rivière de farine de manioc.

Yanawirí ${ }^{65}$ prit cette rivière.

« Jaillissement de Yanawirí,

Étendue de Yanawirí,

Division de Yanawirí. »

On continue ensuite jusqu'à l'embouchure du Kúmuka, le tambour manguaré.

« Jaillissement de Makumuyá ${ }^{\mathbf{6}}$,

Étendue de Makumuyá.

Division de Makumuyá. »

C'est un anaconda, l'esprit [se voit comme] un manguaré.

De l'embouchure du Kúmuka, on continue jusqu'à celle de la rivière Irá « Sang ».

« Jaillissement de Kawanakú,

Étendue de Kawanakú,

Division de Kawanakú. »

En prenant cette rivière, on arrive ensuite à l'embouchure du Mújala.

« Jaillissement de Je'chúmeru Ma'riweló ${ }^{67}$,

Étendue de Je'chúmeru Ma'riweló,

Division de Je'chúmeru Ma'riweló. »

Puis l'on poursuit jusqu'à l'embouchure du Wayuriya ${ }^{68}$.

«Jaillissement de Je'chúmeru Ma'ínaniru ${ }^{69}$,

Étendue de Je'chúmeru Ma'ínaniru,

Division de Je'chúmeru Ma'ínaniru. »

On continue jusqu'aux [autres] lieux de la mère des poissons.

« Jaillissement de Je'chúmeru Junirú,

Étendue de Je'chúmeru Junirú,

Division de Je'chúmeru Junirú. »

« Jaillissement de Je'chúmeru Junirú weló,

Étendue de Je'chúmeru Junirú weló,

Division de Je'chúmeru Junirú weló. »

De l'embouchure de la rivière Sang, on va jusqu'à Ipatuya, rivière de la coca [Quebrada Yupurí] du grand ancêtre des poissons sábalos.

« Jaillissement de l'ancêtre Sábalo,

Étendue de l'ancêtre Sábalo,

Division de l'ancêtre Sábalo. »

On poursuit jusqu'à l'embouchure du Waniyá [Quebrada El Sol].

Je'chúmeru y lava son visage avec des feuilles wiju $^{\mathbf{7 0}}$.

C'est pourquoi l'eau est si bleue. 


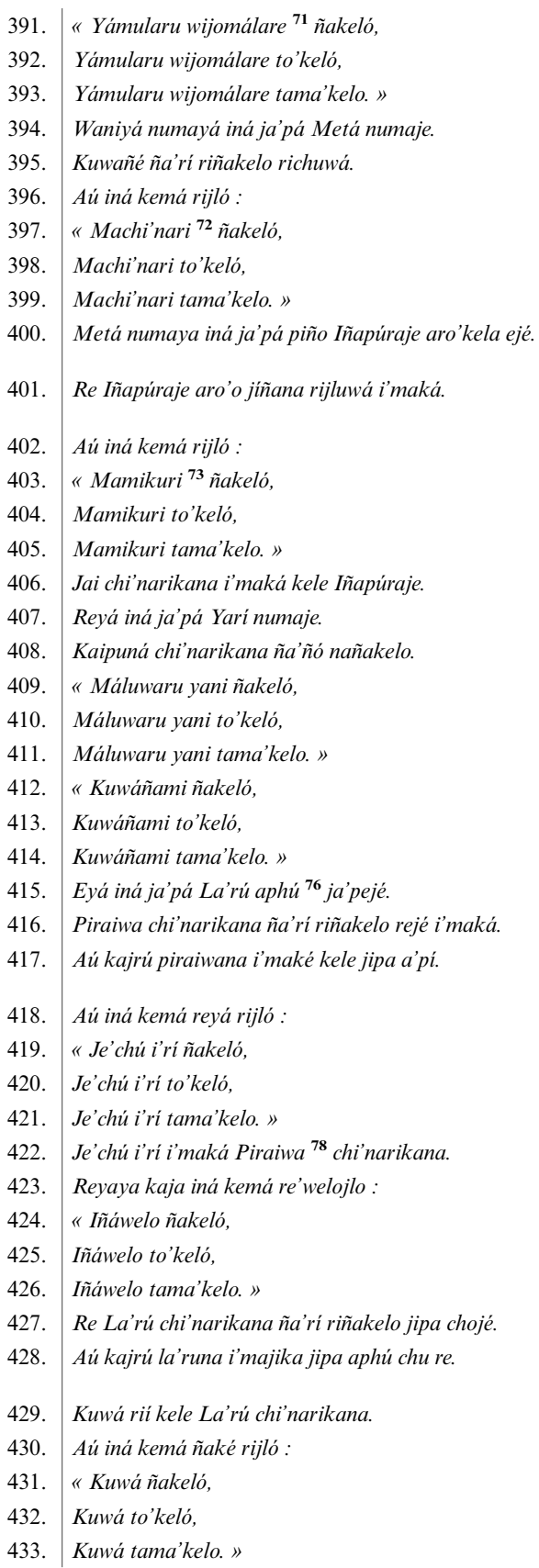

« Jaillissement du wiju de Je'chúmeru,

Étendue du wiju de Je'chúmeru,

Division du wiju de Je'chúmeru. »

De cette embouchure, on passe ensuite à celle du Metá.

C'est le grand singe kuwañé qui prit cette rivière.

Alors on lui dit :

« Jaillissement de l'orphelin Makeratú,

Étendue de l'orphelin Makeratú,

Division de l'orphelin Makeratú. »

De cette embouchure du Metá, on va ensuite jusqu'au bassin de Iñapúraje.

C'est là qu'Iñapúraje y déversait les poissons qu'il puisait.

On lui dit :

« Jaillissement de la guêpe,

Étendue de la guêpe,

Division de la guêpe. »

Iñapúraje était une divinité guêpe.

On va ensuite jusqu'à l'embouchure du Yarí.

Les premiers ancêtres des Karijona prirent cette rivière. « Jaillissement des enfants de Je'rí Máluwaru ${ }^{74}$,

Étendue des enfants de Je'rí Máluwaru,

Division des enfants de Je'rí Máluwaru. »

« Jaillissement de Je'rí Kuwáñami ${ }^{75}$,

Étendue de Je'rí Kuwáñami,

Division de Je'rí Kuwáñami. »

Puis l'on va jusqu'à la rivière du Trou des Aras.

La divinité silure lechero prit cette rivière.

C'est pourquoi beaucoup de ces silures vivaient autrefois sous la falaise [de l'Araracuara ${ }^{77}$ ].

Alors on dit :

« Jaillissement du fils de Je'chú,

Étendue du fils de Je'chú,

Division du fils de Je'chú. »

Cette divinité silure torche était l'un des fils de Je'chú.

On nomme aussi sa sœur [Je'chúmeru] :

« Jaillissement de Je'chúmeru Iñáwelo,

Étendue de Je'chúmeru Iñáwelo,

Division de Je'chúmeru Iñáwelo. »

La divinité Ara s'installa dans la falaise.

C'est pourquoi beaucoup d'aras se nichent dans ses trous.

La divinité Ara s'appelait Kuwá.

Alors on lui dit ceci :

« Jaillissement de Kuwá,

Étendue de Kuwá,

Division de Kuwá. » 


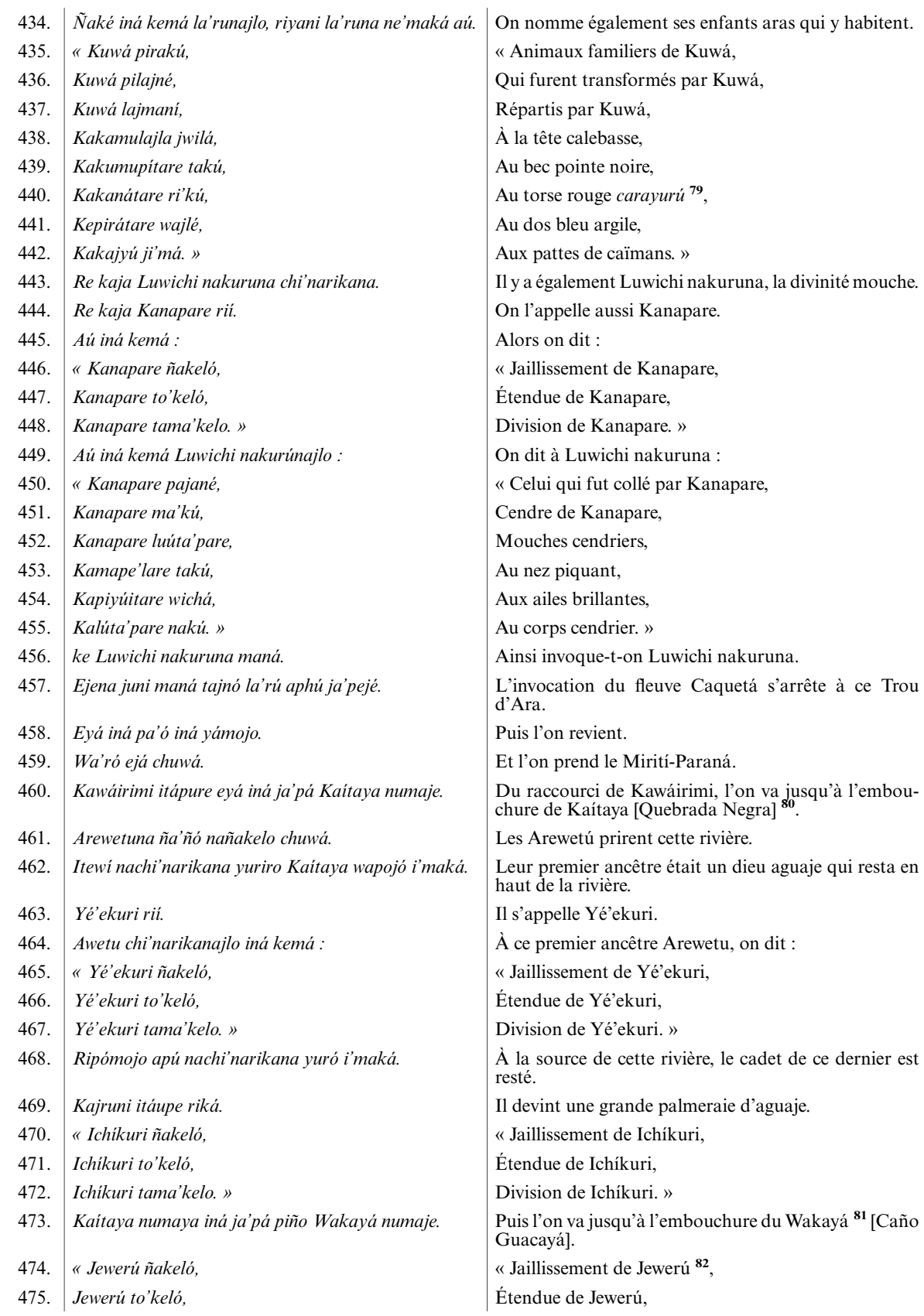




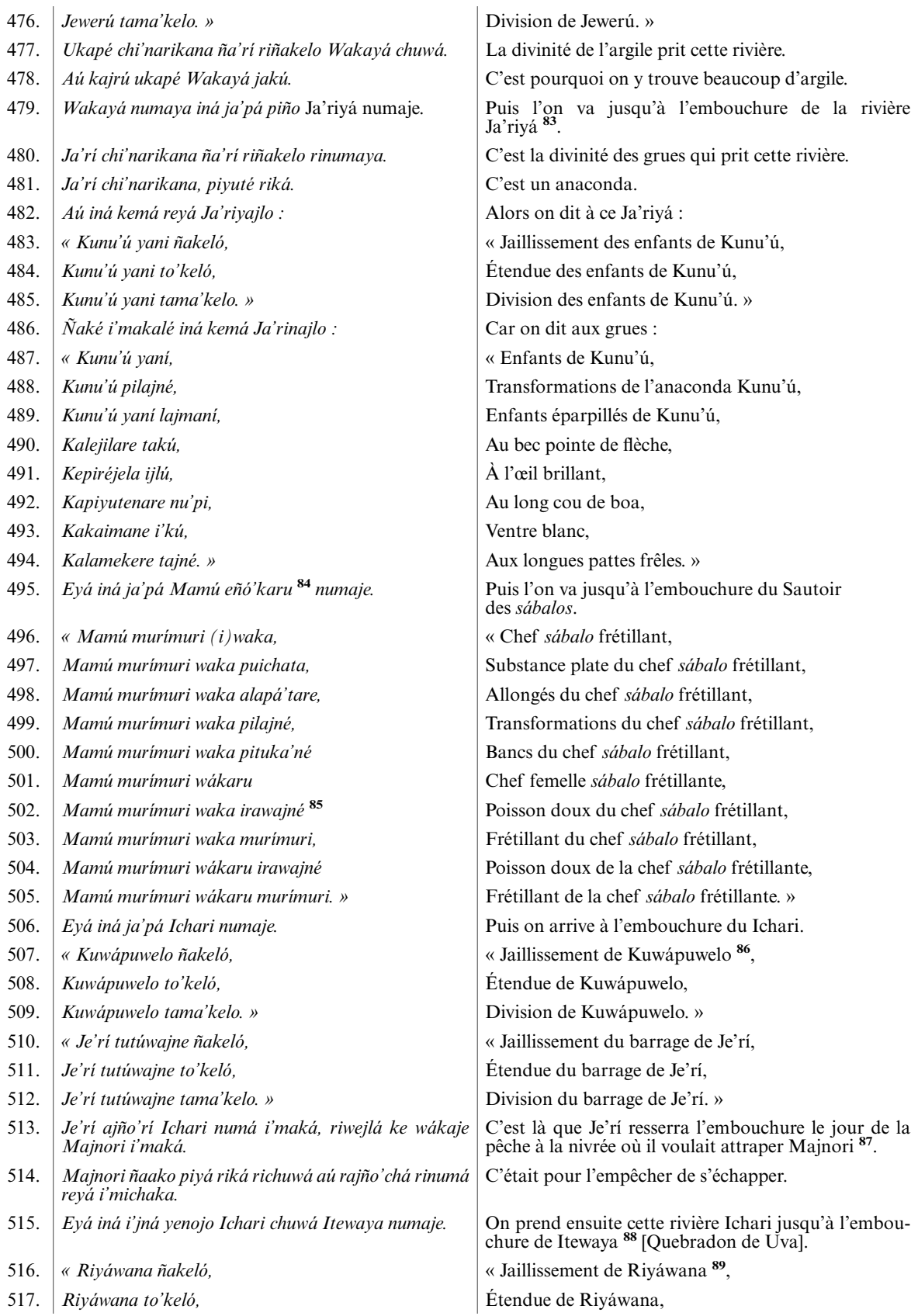




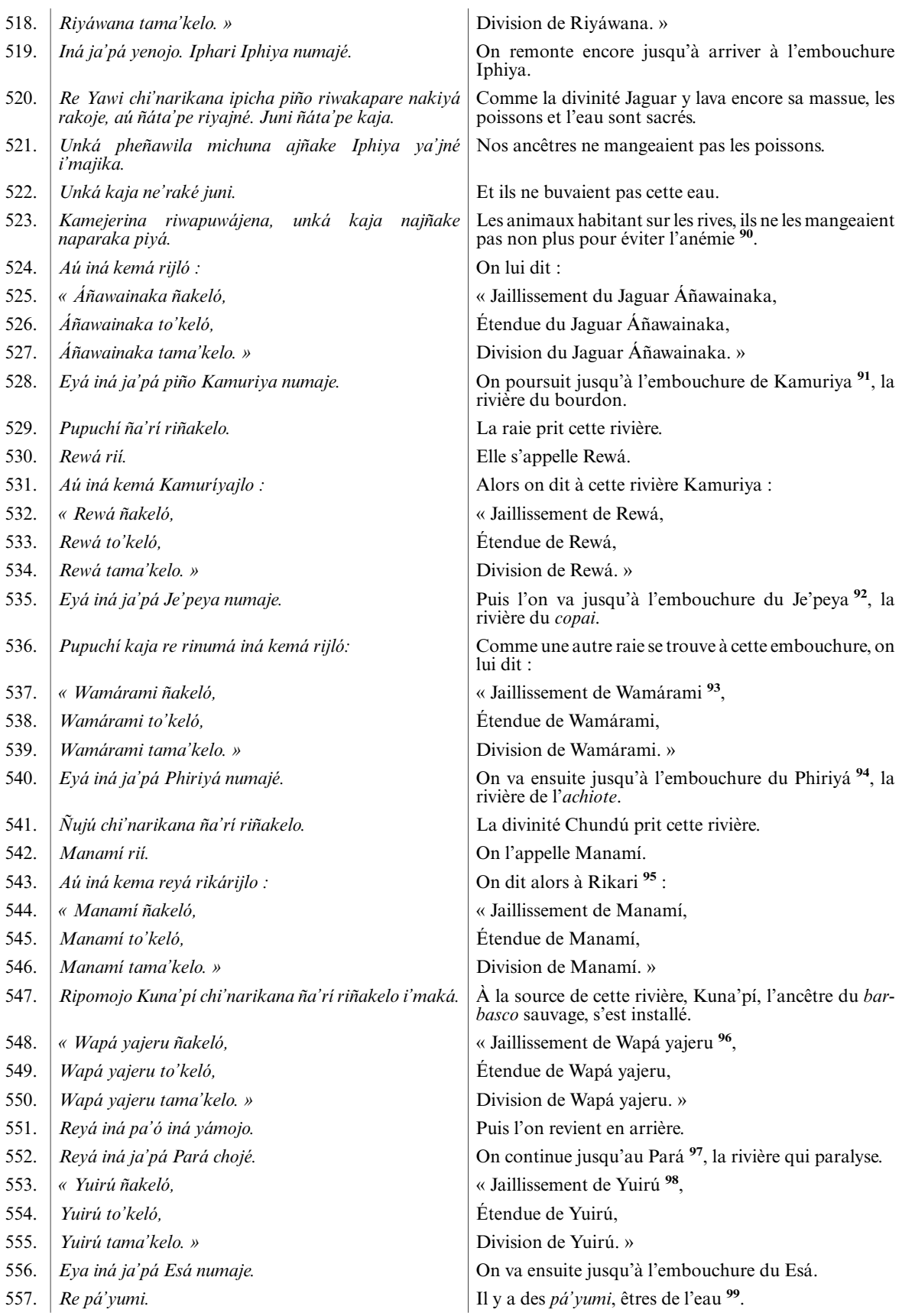




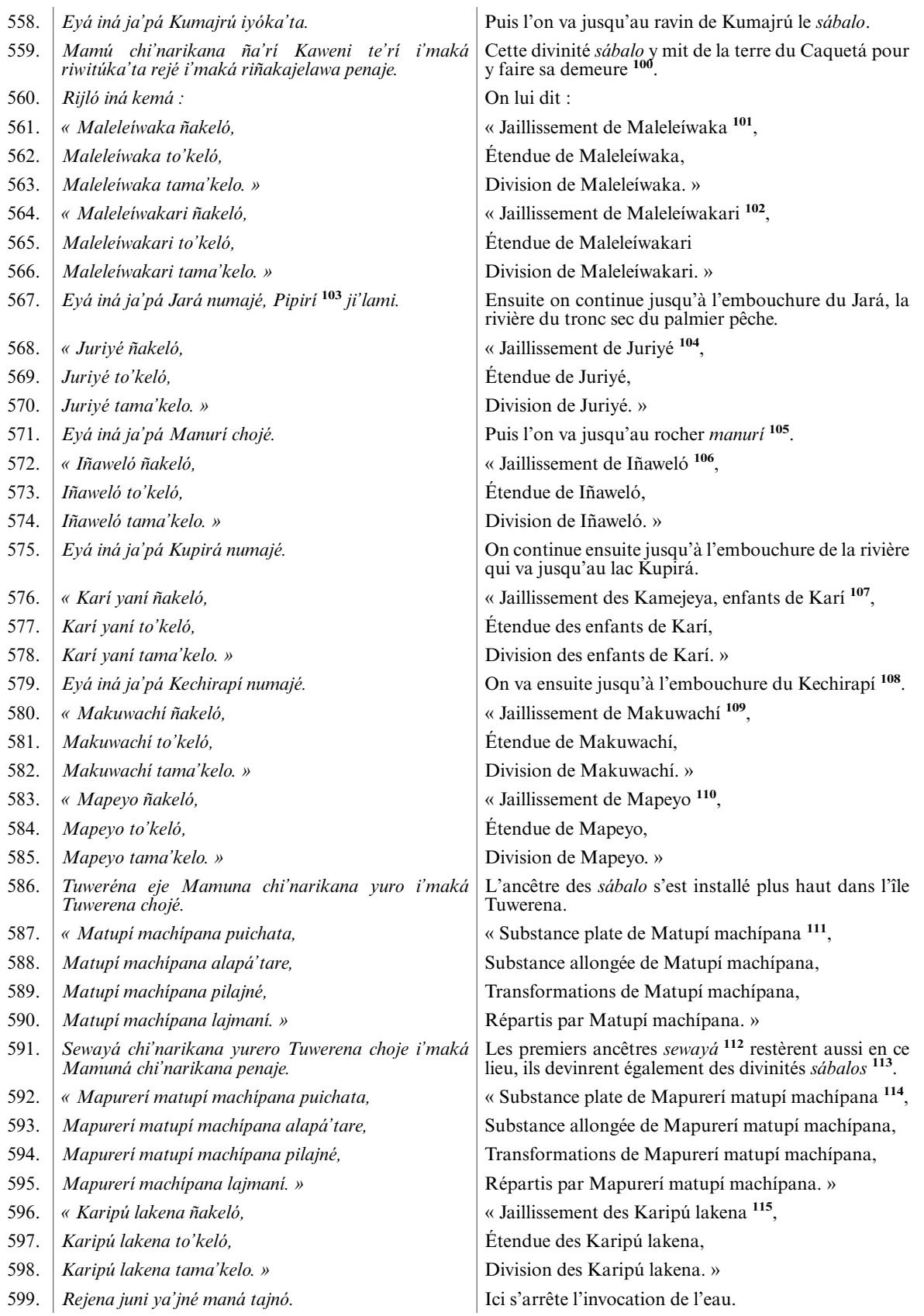


Toutes les incantations des Indiens de langue yucuna ont une structure composée de plusieurs parties enchâssées, souvent de manière assez complexe, les unes dans les autres. On rencontre deux types de vers : des vers invocatoires qui appellent une entité et des vers descriptifs qui la qualifient. Dans les incantations, les vers invocatoires sont fondamentaux, car toute entité doit en priorité être nommée pour pouvoir être manipulée.

Le type d'invocation le plus souvent rencontré est composé de trois vers désignant successivement un fleuve, ou une rivière, par les substantifs ñakelo « jaillissement », to'keló « étendue » et tama'kakelo « division ». Cette invocation forme une matrice comportant une première partie constante et une seconde variable. Ainsi, dans le tableau ci-dessous, v.1, v.2 et v.3 sont les trois vers successifs d'une même strophe invocatoire et $\alpha$ est une constante qui constitue leur partie fixe.

\begin{tabular}{|l|ll|}
\hline v.1 & $\alpha$ & ñakeló, \\
\hline v.2 & $\alpha$ & to'keló, \\
\hline v.3 & $\alpha$ & tama'kakelo. \\
\hline
\end{tabular}

La constante $\alpha$ ajoute des informations permettant d'identifier le lieu selon différents procédés. Les uns donnent les noms (généralement secrets ou réservés à l'incantation) des ancêtres ou créateurs, ou des entités impliquées; les autres y font simplement allusion sans les nommer. Ainsi, $\alpha$ peut désigner un ou plusieurs agents jouissant en quelque sorte d'un " droit » en tant que « propriétaires » ou « maîtres » (paminá) du lieu. Il s'agit de « (grands) ancêtres » ou « divinités » (chi’narikana), le plus souvent en référence à un récit (mythe ou histoire ethnique). Il arrive également que $\alpha$ renvoie à une chose (ancestrale par rapport à l'entité) créée ou possédée par une divinité, ce qui est indiqué par le nom propre (noté NP) de cette dernière, suivi directement par le substantif désignant la chose, par exemple Ro'yé poko (47-49) - Lit. \{NP fosse\} ; Je'chúmeru pujaruka (136-138) - Lit. \{NP [vagin en menstruation]\}. On peut aussi éviter de nommer non seulement les ancêtres ou divinités impliqués, mais encore leurs choses (réputées dangereuses). Dans ce cas, on a comme une invocation dans l'invocation. On évite de nommer non seulement l'entité invoquée, mais encore l'entité ancestrale.

En comparant les vers de l'eau avec les autres vers invocatoires, nous pouvons dégager d'autres caractéristiques plus générales de leur structure. Tout d'abord, ceux-ci sont toujours composés de deux parties. La première partie $\alpha$ de ces vers invocatoires correspond toujours à certains des cas vus avec les appels de l'eau. Il s'agit en l'occurrence d'un ancêtre ou d'un maître de l'entité invoquée, référé soit par son nom propre (parfois noté en plusieurs mots), soit par une formule métaphorique. Cette première partie est répétée à chaque vers de la strophe invocatoire. Quant à la seconde partie, il s'agit toujours d'un substantif qui désigne métaphoriquement l'entité à invoquer en le qualifiant (de par son sens) 
d'une forme particulière. L'ensemble du vers indique alors une relation d'appartenance ou d'origine entre l'entité invoquée et son ancêtre.

\begin{tabular}{|l|l|}
\hline Première partie (constante $\alpha$ ) du vers & Seconde partie (variable) du vers \\
\hline ancêtre de l'entité & entité invoquée \\
nom propre ou proposition nominale & substantif métaphorique de forme \\
\hline
\end{tabular}

Dans la strophe invocatoire de l'ocre (110-112), on trouve pour constante de première partie, le nom de son ancêtre Mañekuni, puis trois variables en tant que substantifs de seconde partie se référant précisément à l'ocre. De même que pour les substantifs ñakelo, to'keló et tama'kakelo, se référant à tout fleuve ou rivière du point de vue yucuna, certains substantifs permettent de désigner métaphoriquement l'ocre. Il s'agit de : pitajné (110), «substance de différentes tailles »; pitakelo (111), « substance amoncelée »; puichata ${ }^{116}$ (112), « substance plate». Ces termes nous donnent, comme pour les désignations métaphoriques de l'eau, une certaine manière de définir et de catégoriser l'entité désignée, en l'occurrence l'ocre.

On trouve un autre exemple intéressant aux séquences 435-442. Après avoir invoqué la rivière de l'Araracuara avec le nom de la divinité ara appelée Kuwá (constante $\alpha$ ), suivie des substantifs métaphoriques de l'eau (431-433), la même divinité est nommée (toujours comme constante) pour désigner " ses enfants » aras, avec cette fois les substantifs métaphoriques spécifiques de cette espèce d'oiseau : pirakú (435), « animaux familiers »; pilajné (436), « substance transformée »; lajmaní (437), « substance répartie ». Ces quelques termes décrivent plus clairement ici les rapports entre la divinité et sa progéniture. Ils indiquent que celle-ci entretient avec eux un rapport maître/animal familier, qu'elle les a créés et éparpillés dans le monde.

Aux séquences 496-505, les poissons sábalos ${ }^{117}$ sont invoqués pour désigner leur «sautoir» (eñó'karu). De nouveau, on se réfère à leur ancêtre sans le nommer. Ce dernier est alors désigné métaphoriquement comme « chef sábalo frétillant » (mamú murímuri waka), de même que sa femelle (mamú murímuri wákaru) ${ }^{118}$. En dehors des termes puichata et pilajné déjà vus, de nouveaux termes métaphoriques désignent leurs descendants sábalos : alapátare (498), « substance allongée »; pituka'né (500), « bancs »; irawajné (502, 504), « poisson doux »; murímuri $(503,505)$, « qui frétille ». On remarque que le terme murímuri peut se rencontrer à la fois dans le terme métaphorique désignant l'ancêtre et dans celui désignant l'entité invoquée $(503,505)$.

Le second type de vers sera appelé ici « vers descriptif ». Ces vers se distinguent des vers invocatoires proprement dits, même si l'on peut considérer qu'ils appartiennent également à la partie invocatoire d'une incantation. Ils ne sont jamais prononcés indépendamment des vers invocatoires. Lorsqu'ils apparaissent, ils constituent le prolongement de ces derniers. Comme les vers invocatoires, les vers descriptifs ont une structure facilement reconnaissable et, surtout, 
un vocabulaire particulier revenant régulièrement pour tous les éléments qui, du point de vue indigène, partagent les mêmes caractéristiques.

La première partie des vers descriptifs est un substantif transformé en adjectif par le préfixe $\mathrm{ka}^{119}$ qui décrit directement une partie corporelle de l'entité invoquée. Le substantif employé désigne toujours un élément de la nature ou une partie de celui-ci. La seconde partie est un substantif désignant la partie corporelle décrite par le premier lexème. Contrairement aux vers invocatoires, aucune partie des vers descriptifs n'est répétée pour une même entité. Toutefois, on peut retrouver exactement la même série de vers descriptifs pour plusieurs entités perçues comme ayant des formes similaires, et donc regroupées et classées dans une même catégorie indigène. À ce titre, la transcription des incantations des Yucuna se révèle être un matériel fondamental pour étudier leurs modalités de classification des éléments de la nature. La structure grammaticale d'un vers descriptif est donc toujours :

\begin{tabular}{|l|l|}
\hline Première partie du vers & Seconde partie du vers \\
\hline $\begin{array}{l}\text { qualification } \\
\text { substantif adjectivisé par } k a-\end{array}$ & $\begin{array}{l}\text { partie corporelle } \\
\text { substantif de partie corporelle }\end{array}$ \\
\hline
\end{tabular}

Contrairement à d'autres invocations riches en vers descriptifs, comme celle des poissons, celle de l'eau présentée ici ne comporte que deux exemples formulés à l'occasion des mentions de deux espèces d'oiseaux impliqués dans l'appel des rivières qu'ils sont censés peupler : les aras (438-442) et les grues (490-494). Les aras, comme les grues, ont été impliqués en invoquant la rivière de leur divinité. De ce fait, ils doivent être invoqués en particulier par des vers invocatoires similaires à ceux qui ont été vus plus haut. Viennent ensuite les vers descriptifs. Reprenons comme exemple la strophe invocatoire des aras étudiée plus haut (435-437). Voici les vers descriptifs qui la suivent :

\begin{tabular}{|l|l|l|l|}
\hline 438 & Kakamujlala jwilá, & ADJ_calebasse_(sphérique) tête & À la tête calebasse, \\
\hline 439 & Kakumupitare takú, & ADJ_pointe_(couleur) noire & Au bec pointe noire, \\
\hline 440 & Kakanátare ri'kú, & ADJ_[peinture végétale]_(couleur) son_torse & Au torse rouge carayurú, \\
\hline 441 & Kepirátare wajlé, & ADJ_argile_(couleur) dos & Au dos bleu argile, \\
\hline 442 & Kakajyú ji'má. & ADJ_caïman patte & Aux pattes de caïmans. \\
\hline
\end{tabular}

Chaque strophe descriptive qualifie les parties qui semblent les plus caractéristiques de l'espèce du point de vue indigène. Dans chaque vers, le second lexème nomme chacune de ces parties selon un ordre qui va de haut en bas dans le sens de la longueur du corps ; en l'occurrence pour un oiseau : de la tête (jwilá) aux pattes (ji'má). En outre, les parties de face (le torse) sont nommées avant celles du dos. Quant à chaque premier lexème, il décrit du point de vue indigène un aspect morphologique de la partie désignée par le lexème qui le suit. Outre le préfixe $k a$ qui adjectivise (noté ADJ) le substantif employé pour décrire chaque partie 
corporelle, un suffixe classificateur est souvent ajouté. Ce suffixe a pour fonction de préciser l'aspect qui est retenu, dans la chose référée par le substantif, pour décrire la partie corporelle désignée. Ainsi pour l'aspect «calebasse » de la tête d'ara (438), le suffixe -la est utilisé en tant que classificateur de sphéricité (déjà vu plus haut avec puicha-la) afin de signaler que c'est la caractéristique sphérique de la calebasse qui est retenue pour décrire la tête de l'ara. Autre exemple, pour l'aspect « argile » du dos, le suffixe -tare signale que c'est la couleur de l'argile (et non pas autre chose) qui caractérise le dos de l'ara (441). Séquence 442, le premier lexème n'a aucun suffixe. On remarque alors que c'est la partie corporelle correspondante de l'animal référé par le substantif, qui est censé composer la partie corporelle désignée. Ainsi, les pattes « caïman » des aras seraient des pattes que ces derniers auraient directement prises au caïman, selon Mario.

Les explications de Mario sont extrêmement précieuses, au point d'être indispensables à la compréhension de ces vers invocatoires. Elles constituent le métalangage d'un indigène sur un langage secret propre à sa culture. Analysons les formes récurrentes de ce métalangage. Elles sont de différents types.

Tout d'abord, une phrase de présentation du point de départ. Elle présente le lieu d'origine d'où part l'invocation de l'eau : «On commence l'invocation de l'eau à partir du delta de l'Amazone ». Mario explique que son commencement verbal correspond à un point de départ topographique. Ce type de phrase est bien sûr unique dans cette invocation, puisqu'elle ne peut avoir qu'une seule origine. Ensuite, on rencontre une phrase d'explicitation de l'invocation, qui annonce que l'on s'adresse à l'eau de ce lieu : « On dit à l'origine du delta de l'Amazone ». Ce qui suppose que cette eau soit une entité apte à écouter les paroles qu'on lui adresse. Ce type de phrase est rappelé avant presque tous les vers invocatoires. Séquence 6, on trouve une phrase descriptive de la forme de l'invocation : « Ils ne sont que trois [vers] ». Ce type de phrase est assez rare dans le matériel recueilli. Il manifeste une préoccupation formelle dans la répétition des vers.

Séquence 7 , nous avons une phrase décrivant un déplacement, en partant du lieu nommé précédemment : "Puis on continue ». Ce type de phrase considère ainsi que prononcer un mouvement revient d'une certaine manière à le réaliser. La propriété performative du langage - où l'action repose sur le fait même d'énoncer cette action - fonctionne ici de manière exemplaire. Ce type de phrase clôt tout commentaire sur une strophe invocatoire d'un lieu, afin de passer au suivant. Séquence 8 , on a ensuite immédiatement le nom du lieu d'arrivée : «On arrive à l'embouchure de Mawiná [l'Amazone] ». Il s'agit du nouveau nom de lieu (noté NL) auquel va s'adresser l'invocation. Il commence tout commentaire sur les strophes d'un nouveau lieu. Il arrive fréquemment que la description du déplacement et l'indication du nom du lieu d'arrivée soient prononcées en une seule phrase constituant à la fois la clôture de l'explication des vers invocatoires précédents et l'initiale de celle qui suit : « On continue jusqu'à l'embouchure du Puré » (22). 
Séquence 23, on a une phrase de révélation de l'acte de l'ancêtre sur le lieu : «La divinité sábalo remonta le fleuve Puré ». Dans ce type de phrase, on peut également nommer plusieurs ancêtres, comme par exemple les Karipú lakena (29). Cette phrase présuppose toujours un droit du maître sur le lieu, reposant sur un certain pouvoir de faire respecter ce droit par sa capacité à sanctionner (en tant qu'être surnaturel), sur le fait d'avoir été le premier à en faire usage (parfois à le créer), et souvent sur sa capacité à être encore censé l'habiter (comme un être immortel). Ce type de phrase dit le plus souvent que l'ancêtre « a pris » le lieu (en premier). Parfois, il s'agit d'un événement mythique fondateur : «C'est là que les Karipú lakena abattirent l'arbre patriarche wa'yuja»(29). Certaines phrases rappellent le passage du mythe où est révélée la transformation en question : « En basculant, il devint l'Apaporis » (30). D'autres phrases résument ce passage du mythe : « Comme Jamerú leur dissimulait [l'eau] à l'intérieur, elle le [l'arbre] fit passer à travers la terre » (32). L'allusion au passé mythique peut expliquer une situation contemporaine, notamment un danger ${ }^{120}$ ou une recommandation : « C'est pourquoi nous disons que cette mère de l'eau est si dangereuse » (33). Plus précisément, nous dirons que le récit mythique est souvent explicatif des règles à appliquer encore de nos jours. Quiconque ignore ou transgresse ces règles peut donc en subir les conséquences néfastes. Par exemple : 130-134 (notamment 132, «Si on mange les poissons, ou si on boit l'eau, on aura des enfants difformes »), 145-162. Parfois Mario apporte une information complémentaire en rapport avec l'une des entités impliquées dans l'incantation, mais ne traitant pas directement des vers invocatoires en question : "Nous avons en commun avec eux un même grand ancêtre anaconda » (92). Telle une visite guidée, l'énumération des cours d'eau faite par Mario permet également de situer des éléments de la nature, généralement évoqués dans la parole mythique : « Il y a un gros rocher » (140).

En suivant ce parcours des différents fleuves et rivières invoqués par les soigneurs de langue yucuna, nous avons tenté de montrer que leurs incantations sont riches en informations sur leur territoire, ainsi que sur la faune et la flore. Elles constituent en quelque sorte des encyclopédies orales du savoir indigène qui posent un véritable défi aux anthropologues et linguistes voulant en rendre compte car leur structure (très imbriquée) est complexe, des connaissances mythologiques sont nécessaires pour leur compréhension, enfin des conditions particulières sont requises pour y avoir accès. Elles sont généralement réservées aux initiés aptes à les mémoriser et, de préférence, aux descendants d'une même filiation patrilinéaire.

Pour les jeunes cherchant à apprendre ces incantations, une difficulté essentielle consiste à répéter de mémoire leurs interminables invocations qui, en raison même de leur dimension encyclopédique, sont censées garantir le respect des conditions de formulation des paroles magiques. En effet, aux yeux des Yucuna, il est impossible de prodiguer des soins chamaniques importants sans réciter ce gigantesque savoir, capacité qui forme une des conditions préalables aux effets 
souhaités des paroles magiques. De plus, il est, selon eux, impensable d'acquérir cette faculté de mémorisation sans respecter un ensemble d'interdits (principalement alimentaires et sexuels) nécessaires pour passer les différents rites d'apprentissage et d'initiation (Yuruparí). Par conséquent, on peut dire que, chez les Yucuna, c'est la sévérité même des conditions de mémorisation d'une telle quantité de savoir qui garantit le respect des conditions d'emploi des paroles magiques. Cela pose un problème pour l'avenir d'un tel savoir. En effet, d'un côté, les jeunes yucuna acceptent rarement de s'astreindre à de telles ascèses pour acquérir ces facultés, alors que, précisément, la société englobante offre en permanence de nouveaux palliatifs au chamanisme (notamment dans les domaines de l'éducation, de la santé et de l'exploitation des ressources naturelles); de l'autre, cette même société englobante est encore loin de valoriser suffisamment ce savoir (ou de lui reconnaître une certaine utilité). Du coup les recherches dans ce domaine ne sont pas développées pour profiter du savoir des derniers soigneurs avant leur disparition et les méthodes traditionnelles d'apprentissage qui existent encore ne sont pas assez renforcées. Il devient donc urgent pour les anthropologues et linguistes d'en rendre compte pour relever ce défi. *

* Manuscrit reçu en novembre 2009, accepté pour publication en juillet 2010.

\section{Notes}

1. Les Yucuna sont généralement définis par rapport à la langue qu'ils parlent habituellement : le yucuna, langue appartenant à la famille linguistique arawak. Ces Indiens sont répartis en cinq «tribus » ou groupes de filiation patrilinéaires (Van der Hammen 1991; Schackt 1994; Fontaine 2008a) auto-dénommés Kamejeya, Je'rúriwa, Jimíkepi, Jurumi et Jupichiya, entretenant entre eux des relations d'alliance exogamique. Autrefois chacun de ces groupes parlait une langue distincte, mais aujourd'hui, après de multiples guerres tribales se terminant par des rapprochements avec les Kamejeya, seule la langue de ces derniers aurait survécu pour constituer la langue yucuna contemporaine. Ces différents groupes de filiation entretiennent une exogamie linguistique avec d'autres groupes voisins, principalement avec les Tanimuca et les Letuama (Jacopin 1972; 1981). Originairement éparpillés autour de l'axe du fleuve Mirití-Parana, entre le Caquetá et l'Apaporis, ces groupes étaient autrefois des chasseurs horticulteurs semi-nomades, mais, depuis l'influence des exploiteurs de caoutchouc (caucheros) du tout début du $\mathrm{xx}^{\mathrm{e}}$ siècle, les Yucuna se sont peu à peu rapprochés des rives de la partie navigable du Mirití-Parana. Depuis le milieu des années 1970, les Yucuna ont tendance à se sédentariser de plus en plus sur le Bas Caquetá à proximité du village de La Pedrera, le centre de l'économie locale (Fontaine 2008a), et à tirer leur subsistance de la pêche. Comme beaucoup d'autres groupes du Nord-Ouest de l'Amazonie, les Yucuna habitent de manière traditionnelle dans de grandes maisons collectives appelées malocas (Jacopin 1972 ; Schackt 1994), ils mâchent de la coca qui occupe une place centrale dans les rituels et les institutions (Fontaine 2008c) et pratiquent le Yuruparí, rite d'initiation où les jeunes hommes jouent des flûtes et des trompes sacrées que les femmes n'ont pas le droit de voir (Mich 1994).

2. Sur les rapports que les Yucuna entretiennent avec leur territoire, voir Jacopin (1972) et Van der Hammen (1991).

3. Ce thérapeute nous fournit ici une version un peu spécifique de ce genre de vers appartenant aux incantations, puisque son langage chamanique comporte de nombreux termes propres aux 
Matapi (auto-dénommés Jupichiya), dont la langue usuelle a aujourd'hui quasiment disparu au profit de la langue yucuna. De nombreux termes sont néanmoins empruntés au yucuna. Dans les notes faisant référence aux termes en langue indigène, les mots proprement matapi réservés à l'incantation seront notés (mat. I), à la différence des mots matapi non réservés aux incantations notés (mat.).

4. Ce type de langage secret ne pouvait absolument pas être recueilli officiellement, c'est-à-dire avec l'accord de la communauté dans laquelle j'ai travaillé. L'interdit de transmission de ces paroles m'a donc contraint à les transcrire également "en secret» sous la responsabilité de Mario Matapi, le soigneur qui nous les a divulguées. Par ailleurs, j'ai également pu enregistrer ces paroles, ce qui fut critiqué par Milciades Yucuna, qui m'avait également dicté en secret des incantations, mais sans que celui-ci ne m'autorise à les enregistrer. Selon ce dernier, cela pourrait déclencher la colère des divinités impliquées et sanctionner non seulement celui qui les écoute, mais surtout celui qui les divulgue.

5. Rappelons que ce rite est connu pour être pratiqué dans le Nord-Ouest de l'Amazonie en l'honneur de la divinité Yuruparí. Les hommes y jouent des flûtes et des trompes sacrées, et apportent dans leur maison collective et cérémonielle des fruits sylvestres en offrande à la divinité (Mich 1994). Durant le rite, les femmes ne sont pas autorisées à voir ou à s'approcher des ritualistes et de leurs instruments, ni même à marcher sur le sol qu'ils ont foulé de leurs pieds. Selon les Indiens, les femmes pourraient mourir de leurs menstruations à la simple vue des trompes sacrées. Les hommes pratiquant le rituel doivent d'ailleurs se soumettre à d'importantes restrictions alimentaires durant de longues périodes et s'abstenir de toutes relations avec les femmes.

6. Juni ji'lami (en yucuna, noté yuc.) : lit. \{eau [manche coupé]\}, " manche coupé de l'eau »; lieu d'origine de l'eau.

7. Lorsqu'une partie d'un mot peut ne pas être prononcée (en langage rapide), nous la mettons entre parenthèses.

8. Mawiná (yuc.) : Me’taká (en langue matapi d'incantation, noté mat. I) ; Amazonas (en espagnol, noté esp.) ; l'Amazone.

9. Sulimó (yuc.) : Pajurí (en langue matapi, noté mat.) ; Solimões (en portugais brésilien, noté br.).

10. Kawemí (mat.) : Wépuru (mat. I) ; Kamaweni (yuc.) ; Caquetá (esp.).

11. Makera'pá (mat. I) : divinité poisson Sábalo ; Brycon melanopterus (nom scientifique, noté sc.).

12. Wa'yuja (yuc.) : palo pelusa (en espagnol vernaculaire, noté esp. ver.) ; arbre (non identifé) ; on dit que « ses branches ne brûlent pas bien » (kawirí riná).

13. Comme Jamerú avait attaché cet arbre au ciel par une liane, l'arbre fut projeté dans le ciel en tournant (comme un pendule) et en versant son eau partout dans le monde. Ensuite Lajmuchí se transforma en écureuil pour ronger le lien du second arbre à eau, mais Jamerú fit un sortilège pour qu'il passe à travers la terre (jusqu'au monde tellurique), au moment où les Karipú lakena l'abattirent. Cet extrait du mythe des Karipú lakena diffère de la version de Milciades que nous avons transcrite par ailleurs car, dans cette dernière, Jamerú leur cache l'arbre Weí (du delta de l'Amazone), tandis que l'arbre Wa'yuja, également abattu par les Karipú lakena, était chaque nuit rebouché par la fille de Je'chú, Mananiyó (il semble que ce soit l'inverse pour Mario).

14. (J)ero’jopha (yuc.) : mocho dormilón (esp. ver.) ; traíra (br.) ; ro’yé (mat.) ; ro’yéa (en tanimuca, noté tan.) ; Hoplias malabaricus (sc.) : poisson qui donna son nom au fleuve Taraira (yuc. ero’jópaya), terme d'origine tupi (nheengatu).

15. Poko (yuc.) : pozo (esp.); fosse fluviale.

16. Itáupe (yuc.) : cananguchal (esp. ver.); palmeraie d'aguaje ; Mauritia flexuosa (sc.).

17. Karikaya (mat.) : ce terme signifie tucupi, sauce à base de manioc ; Lac Caparú (esp.). Le nom colombien de ce lac fut donné récemment par des biologistes ayant installé leur station biologique. Il reprend le mot yucuna pour signifier «singe laineux » Lagothrix lagothricha (sc.), en raison de leur grand nombre à proximité du lac.

18. Iyáruri (mat. I) : Mojocoy (esp. ver.); ancêtre tortue terrestre des Macuna.

19. Aiyapa (mat. I) : Picón (esp. ver.); ancêtre toucan. Ses descendants sont, comme leur nom l'indique, les Tucano. 
20. Ukatekuwa (mat. I) : ancêtre d'un arbre (non identifé) qui ressemble au laurier (esp. ver. : laurel). On en tire de l'encens (yuc.: naika). Les Karipú lakena utilisèrent cet encens pour faire « revivre » des gens. Les soigneurs disent qu'il contient de «l'esprit de cœur».

21. Makuwachi (mat. I) : Po'peya (yuc.) ; nom du grand ancêtre anaconda. Les Letuama seraient sortis du milieu de son corps.

22. Mañekuni (mat. I) : Jewa (yuc.); divinité de l'ocre.

23. Yáweka (mat. I) : grand ancêtre de l'acouchi; Myoprocta acouchy (sc.).

24. Jáñawainaka (mat. I) : nom tribal des divinités jaguar. Ici, il s'agit du méchant oncle jaguar de Jiyánama (mythe de Jiyánama).

25. Jiñáñapa (yuc.) : olla de picalón (esp. ver.) ; « marmite à poissons-chats », Pimelodella sp. (sc.).

26. Le poisson-chat a des pointes à chaque « bras » [ou nageoire].

27. Mario dit que, de nos jours, plus personne n'a ni la force, ni le courage d'antan pour s'affronter ainsi sur le rocher.

28. Wana (yuc.) ; guaulo, bambú (esp. ver.) : ces bambous sont aussi utilisés pour faire les bâtons de rythme de certaines danses.

29. Jíwaru (mat. I) : l'un des noms de Je'chúmeru, la mère des poissons, voir le mythe de Kanumá (Fontaine 2010b).

30. Jíwa náneru (mat. I) : l'un des autres noms de Je'chúmeru.

31. Mario rappelle que Yuwinata, la terre d'origine des Matapi, est proche de cet endroit, puisqu'elle se situe entre les deux rivières Kunami et Ke'chirapí.

32. Jatímaja (yuc.) : nom d'un clan aîné des Tanimuca, aujourd'hui disparu.

33. Jañunaké kapiri (mat. I). Il s'agit du Jaguar Jiyánama (appelé aussi Iyánama ou (J)iyánuma) qui est ici nommé à deux reprises : la première fois avec son premier nom Jañunaké, la seconde avec ses deux premiers noms Jañunaké kapiri.

34. Weipa, Yarumákaru, Wiyanumá (mat. I) : il s'agit des trois noms donnés au même « jaguar d'eau » (tigre de agua), aussi appelé en yucuna : junijimani.

35. Suye (yuc.) : enfants surnaturels habitant dans l'eau de la rivière. Ils ont la particularité de gonfler subitement pour devenir gigantesques dès que l'on essaie de les attraper.

36. Mario fait référence à l'histoire célèbre du bal des poissons qui, selon lui, serait originaire des Arewetú (une tribu aujourd'hui disparue) : l'histoire du coffret des poissons qui apparut à une jeune fille au moment de ses premières règles (Jiñana maranare ya'taro pajluwajaru inanarú a'takewarujlo yukuna). Un jour, la jeune fille trouva en allant se baigner dans la rivière Kunami un coffret rempli de plumes de danse. Elle alla prévenir sa famille, mais ses oncles ne parvenaient pas à l'attraper car, à chaque tentative, la boîte plongeait au fond de la rivière. Comme la petite était la seule à pouvoir la toucher, elle l'attacha, et ils purent ainsi s'en emparer. Un étranger (qui était en fait un poisson transformé en humain) recommanda de ne laisser que les hommes adultes toucher à ces plumes, mais les Kejepu ne suivirent pas son conseil. Comme il y en avait beaucoup, tous, femmes et enfants compris, se parèrent de ces plumes pour danser. Alors, durant la nuit de leur danse, une pluie torrentielle fit monter le niveau de l'eau sans qu'ils s'en rendent compte, et ils furent tous noyés.

37. Wa'kuní (mat. I) : suye (yuc.) ; divinité enfant d'eau.

38. Les Kejepu adultes, à la différence de leurs enfants, se convertirent en poissons.

39. Yárau (mat. I) : pensamiento de hueso (esp. ver.). Il s'agit de ce palmier aguaje qui, dans le monde spirituel, est censé être l'os du grand ancêtre matapi appelé Wájlina.

40. Kuna'pí (yuc.) : barbasco de monte (esp. ver.). Plante utilisée pour asphyxier les poissons dans la pêche à la nivrée, Lonchocarpus Nicou DC. spp. (sc.).

41. Ma'parena (mat. I) : divinité barbasco sauvage.

42. Ñamí wayuma (mat. I) : lit. \{[poudre de nuit] jaguar\}, « poudre de nuit du jaguar ». La nuit est censée être obscurcie par une poudre magique. Cette divinité est probablement un jaguar noir.

43. C'est la colline qui fait face au village de La Pedrera, de l'autre côté du fleuve Caquetá (réserve indigène de Comeyafú). On dit que ce lieu est peuplé de nombreux esprits yuruparí, c'est pourquoi on entendrait, lorsqu'il y a du vent, le chant du Yuruparí. 
44. Selon Mario, le Yuruparí serait, comme il est dit ici, le fils aîné de Je'chu. Pour Milciades Yucuna, il serait le fils de Waruwachí qui apparaît dans le mythe de Kari i'rimi. Pour des versions résumées de ce mythe, voir Van der Hammen (1991) et Robayo (2007, p. 106).

45. Le second fils de Je'chú serait un autre Yuruparí.

46. Yáwi jwílaromi (yuc.) : « crâne du Jaguar ». Non loin de La Pedrera, il s'agit d'un rocher ayant la forme d'une tête de jaguar que l'on aperçoit sur la gauche en remontant le fleuve par bateau.

47. On retrouve le nom tribal des divinités jaguars auxquelles appartenait l’oncle de Jiyánuma. Celui-ci était un jaguar qui tua beaucoup de gens en dépit des recommandations de son frère aîné. Il finit par être tué par les hommes qui jetèrent son corps dans le fleuve (mythe du Jaguar ancestral).

48. Kawáwiri et son frère Imarí sont les maîtres du chant de pupurá. Leur histoire dit qu'ils se seraient battus au-dessus de la planche de danse (yuc. : puru; esp. : tablón).

49. Jamerú, la tante des Karipú lakena, habiterait sous les rapides de Córdoba (mythe des Karipú lakena; mythe de Kawáirimi). Tous ceux qui se noient dans ces rapides sont censés aller vivre et travailler pour elle dans son monde.

50. Pichaní, le Tonnerre, est aussi le maître du curare, ce qui expliquerait pourquoi l'on rencontre beaucoup de plantes à curare (yuc. : ja'pá) à proximité de cette rivière. L'actuel resguardo (réserve indigène) où elle se trouve est appelé Curare.

51. Wáipuru (mat. I) : divinité ou ancêtre des pierres de foudre. Mario dit que ce sont des pierres rouges que l'on rencontre sur les plages. D'après lui, elles éclatent quand elles ont été touchées par la foudre. Il est probable que ces pierres soient des fulgurites, c'est-à-dire des morceaux de verre, formés par les impacts de foudre dans le sable.

52. Itapu (yuc.) : brazuelo (esp. ver.). Bifurcation servant de raccourci pour éviter de suivre les larges méandres d'un fleuve lorsque le niveau des eaux est haut. Pour ce raccourci de Kawáirimi, il s'agit d'une bifurcation du Caquetá débouchant sur le fleuve Mirití. Kawáirimi l'aurait fait avec Kanumá pour rattraper les Ñamatu dans leur fuite. Voir le mythe de Kanumá (Herrera Angel 1975 ; Fontaine 2010b).

53. Imariweló (mat. I) : l'un des multiples noms de Je'chúmeru. Elle porte souvent un nom spécifique en tant que mère des poissons d'une rivière particulière.

54. Selon Mario, Makuwaí et Merayú seraient deux grands ancêtres des Miraña, probablement des ancêtres animaux permettant de distinguer leurs clans.

55. Mario dit que les Miraña mâchent la coca en utilisant une espèce de petite noix de coco (yuc. : tuphi).

56. Juni numari (yuc.) : « celui qui est venu de l'embouchure de l'eau ». On dit que, de cette région, seraient venus la divinité aigle harpie et, aussi, beaucoup d'autres gigantesques animaux, ancestraux ou divins.

57. Mythe des Karipú lakena, épisode de l'aigle suprême.

58. Jipaji (mat.) : íji (yuc.) ; urine.

59. Lainúiri (mat. I) : nom du grand ancêtre Jarechina.

60. Perikana (mat. I) : nom d'un grand ancêtre La'yana.

61. Kurerí (mat. I) : nom d'un autre grand ancêtre La'yana.

62. Wapheno (mat. I) : nom de Pá'yumi, le maître de l'eau (mythe des Karipú lakena).

63. Pá'yumi apu (yuc.) : passage de Pá'yumi. Il s'agit d'un passage secret que seuls les chamanes seraient aptes à emprunter grâce à leurs pouvoirs particuliers de déplacement.

64. U’wiyá (yuc.) : du terme u'wí ou « farine de manioc ». Comme dans beaucoup d'autres noms de rivière, le suffixe -yá signifie « rivière ».

65. On se réfère ici au mythe de Yanawirí, l’homme qui, après avoir mangé les poissons de la « lune sanglante ", perdit ses membres et même son tronc, jusqu'à ne plus devenir qu'une tête encore en vie. Au début, il s'enracine sur l'épaule de son frère, puis après que ce dernier ait réussi à s'en débarrasser, il finit par s'installer définitivement sur le dos d'un tapir.

66. Makumuyá (mat. I) : Kúmuka (yuc.) ; divinité anaconda appelée manguaré (esp. ver.). Le manguaré est une paire de grands cylindres de bois (troncs évidés) que l'on martèle sur leurs longueurs 
comme des xylophones. Chaque mélodie rythmée est un message codifié transmis aux populations environnantes.

67. Ma'riweló (mat. I) : Je'chúmeru. Celle-ci y aurait laissé ses enfants poissons.

68. Wayuriya (yuc.). En remontant cette rivière, puis en prenant un sentier en forêt, on arrive à l'ancien lieu de Campoamor (Fontaine 2008b, pp. 136-197).

69. Ma'ínaniru (mat. I) : autre nom de Je'chúmeru.

70. $W i j u$ (yuc.) : feuille (non identifée) utilisée par les jeunes filles pour se graisser le visage et ainsi le rendre plus brillant lors de leurs premières menstruations. Celles-ci sont appelées par Mario primer arroyo («premier ruisseau») au lieu de primer desarrollo («premier développement»), courant en espagnol.

71. Yámularu wijomálare (mat. I) : lit. \{NP [wiru_POSS]\}, «feuille wiju de Yámularu ». Yámularu est le nom de Je'chúmeru pour cette rivière.

72. Machi'nari (yuc.) : orphelin ; lit.\{[Sans_parent_SUBS]\}, «sans parent». Ici il s'agit de Makeratú, le fils orphelin d'un singe géant kuwañé tué par les hommes.

73. Mamikuri (mat. I) : jai (yuc.) ; la guêpe (mythe de Kawáirimi, voir Jacopin 1981).

74. Máluwaru (mat. I) : Je'rí (yuc.) ; grand ancêtre des Carijona. Il est aussi celui qui mangea Mananiyó (fille de Je'chú) et tenta de tuer le fils qu'elle eut avec la Lune (mythe de Majnori).

75. Kuwáñami (mat. I) : Je’rí (yuc.) ; ce terme désigne Je’rí comme un « jaguar ».

76. La'rú aphú (yuc.) : lit. \{ara trou\}, « les trous des Aras » (lieu appelé Araracuara en espagnol). Il désigne une gigantesque falaise dans laquelle viennent se nicher de nombreux aras.

77. Comme l'Araracuara est devenu un lieu de peuplement et donc de pêche relativement important surtout depuis le milieu des années 1970, les grands silures s'y sont raréfiés.

78. Piraiwa (yuc.) : lechero (esp. ver.); ordre des siluriformes, la torche, Brachyplatystoma filamentosum (sc.).

79. Le carayurú (yuc. kena) est une peinture végétale produite à partir des feuilles d'une certaine plante (non identifée). Les Yucuna se la procurent généralement auprès des Macuna. Elle est utilisée dans certains soins chamaniques.

80. Kaítaya (yuc.) : quebrada negra (esp. ver.), ce qui signifie la « rivière noire ».

81. Wakayá (yuc.) : Caño Guacayá (esp. ver.).

82. Je'weru (yuc.). La divinité de l'argile. Mario dit qu'il s'agit de Je'chúmeru et/ou Jamerú. Peut-être hésite-t-il entre les deux.

83. Ja'riyá (yuc.) : lieu où se trouvent l'internat et le corregimiento (centre administratif de l'État) du Mirití.

84. Mamú eñókkaru (yuc.), Brincadera de los sábalos (esp. ver.) : « Sautoir des sábalos ». Ce lieu est plus connu en espagnol sous le nom de Chorro de Tequendama, c'est-à-dire chute de Tequendama.

85. Irawajné (yuc.) : transformation du sang de l'eau. Il s'agit du pez dulce (esp.), soit « poisson doux », Hoplerythrinus unitaeniatus (sc.).

86. Kuwápuwelo (mat. I) : Je'chúmeru.

87. Cela fait référence au mythe de Majnori, le fils de la Lune et de sa sœur Mananiyó (Van der Hammen 1991 ; Robayo 2007, p. 72).

88. Itewaya (yuc.) : rivière du petit poisson itewa.

89. Riyáwana (mat. I) : nom de Je'chú lorsqu'il créa à cet endroit beaucoup d'uva, Pourouma sapida (sc.). C'est pourquoi on appelle aussi cette rivière quebradon de uva (" rivière de l'uva »).

90. Aparákaje (yuc.) : devenir anémique. On dit que cela donne une maladie du sang qui « rend jaune » et paralyse en gênant les mouvements et la respiration.

91. Kamuriya (yuc.): "rivière du bourdon» (quebradon del abejon); du nom du bourdon kamuyari.

92. Je'peya (yuc.) : « rivière du copai». Terme dérivé de je’pé (esp. ver. copai) qui est un bois résineux utilisé pour faire des torches.

93. Wamárami (mat. I) : nom de cette raie. 
94. Phiriyá (yuc.) : terme dérivé de phirí, le roucou (esp. ver. achiote ; sc. Bixa orellana), qui signifie « rivière du roucou ». Le roucou est un fruit à graines rouges très colorantes utilisées comme peinture pour le corps ou l'artisanat.

95. Rikari est le nom de la divinité Chundú. Certains êtres disposeraient du dangereux pouvoir du chundú (qui rend follement amoureux) : hocco, ara, amazone farineuse, daguet, singe géant kuwañé, démons et, même, le caimillo (yuc. je'pela), Pouteria sp. (sc.). Mario dit que c'est lié aux os des morts (yuc. warumí) qui ne peuvent pas être touchés, surtout par celui qui les a tués.

96. Wapá yajeru (mat. I) : nom de cet ancêtre du barbasco sauvage (esp. ver. barbasco de monte).

97. Pará (yuc.) : rivière sacrée qui, à l'instar d'Iphiya, inflige l'anémie et la paralysie ; terme dérivé d'Aparákaje. Je'chúmeru y aurait peut-être lavé son vagin en menstruation comme pour le Ináwaya.

98. Yuirú (mat. I) : nom de Je'chúmeru.

99. Mario avait manqué de nous indiquer cette rivière. Il dit qu' « il n'y a rien », c'est-à-dire pas d'incantation ou de danger particulier pour la consommation de l'eau et des animaux, mais qu' « il y a des pá'yumi », des êtres de l'eau qui sont réputés dangereux et qui sont les ancêtres des Kamejeya. Il est possible qu'il n'ait pas voulu nous en dire plus.

100. Cela explique pourquoi la terre y est la même que sur le Caquetá. C'est aussi l'ancien lieu de Campoamor.

101. Maleleíwaka (mat. I) : nom de cette divinité sábalo.

102. Maleleíwakari (mat. I) : nom de l'épouse de Maleleíwaka.

103. Pipirí (yuc.) : chontaduro (esp. ver.); palmier pêche, Bactris gasipaes (sc.).

104. Júriye (mat. I) : nom de l'ancêtre du palmier pêche. Selon Mario, il aurait aujourd'hui l'apparence d'un « tronc mort» ou « sec » (yuc. ji'lami) de ce palmier.

105. Manurí (yuc.) : rocher qui gêne la progression par canoë sur le Jará.

106. Iñaweló (yuc.) : nom de la « mère des poissons », soit Je'chúmeru.

107. Les enfants (ou descendants des enfants) de Kari sont les Kamejeya.

108. Sur les bords de cette rivière Kechirapí se sont installés jadis les premiers Matapi (Jupichiya) à Yuwinata (Matapi et Matapi 1997).

109. Makuwachí (mat. I) : ancêtre boa des Matapi.

110. Mapeyo (mat. I) : autre ancêtre des Matapi, cadet du premier.

111. Matupí machípana (mat. I) : nom de ce grand ancêtre sábalo.

112. Sewayá (yuc.) : petit poisson ressemblant d'après Mario au bocachico, Prochilodus sp. (sc.).

113. On dit qu'ils prennent parfois une apparence humaine, mais ce sont des poissons.

114. Mapurerí machípana (mat. I) : nom des ancêtres sewayá.

115. D’après Mario, pour se référer à la dernière source du Mirití-Parana, il faut terminer l'incantation en nommant les Karipú lakena, car ils furent les premiers à passer par là.

116. Il s'agit du même terme utilisé pour désigner la massue plate (126-128) du jaguar dont il a été question un peu plus haut. On peut donc utiliser ce terme aussi bien en première partie constante d'un vers, qu'en seconde partie variable.

117. Mamú (yuc.) : sábalo (esp. ver.) ; Brycon melanopterus (sc.), voir Galvis et al. (2006, p. 190).

118. - ru est un suffixe marquant le féminin.

119. Le préfixe $k a$ - devient $k e$ - devant la racine d'un lexème en - $e$ ou - $i$. Ce préfixe qui change le lexème en adjectif est connu comme caractéristique de la plupart des langues de la famille linguistique arawak.

120. Jamerú est considérée comme dangereuse notamment près des rapides de Córdoba, où elle est censée habiter. Ce serait elle qui ferait disparaître de nombreuses personnes dans ces rapides. Par ailleurs, elle est particulièrement mentionnée dans les invocations où il est question de se prémunir de ses malédictions : par exemple, lors de l'incantation de préparation du lieu de la maloca que l'on souhaite construire. 


\title{
RÉFÉRENCES CITÉES
}

\begin{abstract}
FONTAINE Laurent
2008a Paroles d'échange et règles sociales chez les Indiens yucuna d'Amazonie colombienne, L'Harmattan, Paris.

2008b Récits des Indiens yucuna de Colombie. Texte bilingue, L'Harmattan, Paris.

2008c «Les monnaies chez les Yucuna d'Amazonie colombienne : de la coca à mâcher au peso ", in Éveline Baumann, Laurent Bazin, Pépita Ould-Ahmed, Pascale Phelinas, Monique Sélim et Richard Sobel (éd.), L'argent des anthropologues, la monnaie des économistes, L'Harmattan, Paris.

2010a "Agents" ou "patients"? Les chamanes yucuna d'Amazonie colombienne », in Aurore Monod Becquelin et Valentina Vapnarsky (éd.), L'agentivité. Ethnologie et linguistique à la poursuite du sens, Ateliers du LESC, 34, Nanterre [http://ateliers.revues.org/8229, consulté le 30/10/2010].

2010b « Histoire de Kanumá », in Boyd Michailovsky, Michel Jacobson et Séverine Guillaume, Archive du Lacito et Centre de ressources pour la description de l'oral (CRDO) du CNRS. [http://lacito.vjf.cnrs.fr/archivage/tools/show_ text.php?id=crdo-YCN_KEYAKO_SOUND, consulté le 23/04/2010].
\end{abstract}

Galvis Germán, José Iván Mojica, Santiago R. Duque, Claudia Castellanos, Paula SÁnchez-Duarte, Mariangeles Arce, Ángela Gutiérrez, Luz F. JimÉnez, Marisol Santos, Silvia Vejarano, Fernando Arbeláez, Edgar Prieto, Mauricio Leiva

2006 Peces del medio Amazonas. Región de Leticia, Conservación Internacional, Bogota.

Herrera Angel Leonor

1975 «Kanuma : un mito de los Yukuna Matapi », Revista colombiana de Antropología, 18, pp. 383-415.

JACOPIN Pierre-Yves

1972 «Habitat et territoire yucuna », Journal de la Société des Américanistes, 61, pp. 107-139.

1981 La parole générative de la mythologie des Indiens Yukuna, thèse de doctorat, université de Neuchâtel, Neuchâtel.

Matapi Carlos et Uldarico MataPI

1997 Historia de los upichia, Tropenbos, Bogota.

Mich Tadeusz

1994 "The yuruparí complex of the Yucuna indians : the yurupari rite », Anthropos, 89 , pp. 39-49.

Robayo Camilo

2007 Auffassung der Person "inau'ké » und des " pensamiento » bei den YukunaMatapí, thèse de doctorat, Philipps-Universität Marburg, Marbourg. 


\section{SCHACKT Jon}

1994 Nacimiento yucuna. Reconstructive ethnography in Amazonia, thèse de doctorat, Université d'Oslo, Olso.

VAN DER Hammen Maria Clara

$1991 \quad$ El manejo del mundo, Tropenbos, Bogota. 